ABSTRACT

12

\title{
Towards the maximum efficiency design of a perovskite solar cell by material properties tuning: A multidimensional approach
}

\author{
1 Electrical Materials Laboratory (LAMATE), Department of Electrical Engineering, Universidade Federal de Santa Catarina (UFSC), Florianópolis \\ 88040-970, SC Brazil \\ 2 Fotovoltaica UFSC - Solar Energy Research Laboratory, Universidade Federal de Santa Catarina (UFSC), Florianópolis 88056-000, SC Brazil. \\ Manfred G. Kratzenberg ${ }_{1,2 *}$, Ricardo Rüther2 \& Carlos R. Rambo1
}

In order to obtain significant increases in the Power Conversion Efficiency (PCE) of solar cells, we argue that future cell research and development should be based on the concomitant improvement of multiple material properties, rather than on the state-of-the-art one- or two-dimensional improvements. In this context, researchers should know, which combined material properties and cell design parameters lead to the highest efficiency increase. For the same objective, it should also be known which relationships in-between these variables have to be adjusted. Such knowledge becomes available by simulation and numerical optimization, which we present for a Perovskite Solar Cell (PSC) in a hypercube space of variables. We prove that its PCE increases principally because of the nonlinearities of this model in the multidimensional space, elucidating the importance of the concomitant variable improvement in the PSC optimization. In the most optimal case, efficiencies of at least $27.6 \%$ can be obtained by the simultaneous improvement of the cell's material properties, and light trapping, for a large range of absorber layer thickness of $t_{0}=160 \ldots 400 \mathrm{~nm}$. The lower thickness value results in a significant reduction of the cell's lead $(\mathrm{Pb})$ content.

Keywords: Perovskite solar cells, Simulation, Numerical optimization, Light trapping, Cell designs.

* Corresponding author.

E-mail address: manfredkratzenberg@gmail.com (M. Kratzenberg)

\section{Introduction}

Early research in hybrid solar cells (Huang and Huang, 2014; O’Regan and Grätzel, 1991; Wong et al., 2007) led to the concept of the so-known PSC (Kojima et al., 2009), a hybrid thin-film cell, which absorber layer is constituted by an organic-inorganic compound of semiconductor materials. In searching for new materials and properties of this cell, more recent advances provided the steepest

PCE increase in comparison to all other types of cells, reaching the state-of-the-art efficiency of 
$124.2 \%$ for a manufactured prototype (NREL, 2019). Several attempts were accomplished in order to

2 reduce the toxicity inherent to the heavy-metal $\mathrm{Pb}$, present in the absorber layers of high-efficiency

3 PSCs. Such methods consider the complete (Devi and Mehra, 2019; Dixit et al., 2019), or partial

4 substitution of this element (Liu et al., 2018). However, they lead in much lower PCE, if compared to

5 the state-of-the-art efficiency. Our objective is to provide more knowledge and insights about the

6 optimization process of perovskite solar cells and propose high-efficiency designs for future PSC

7 developments. To access this knowledge, and the design concepts, we propose a multidimensional numerical optimization of a cell's drift-diffusion model. Our design concepts show, that the state-ofthe-art PCE can be significantly increased, while the $\mathrm{Pb}$ content can be reduced up to an order of magnitude.

\section{Optimization of perovskite solar cells}

13 State-of-the-art PSCs are mostly optimized based on cell prototyping, where the cell's efficiency is

14 increased in small steps as a function of one or two different variables, as permitted by graphical 15 visualization in one- or two-dimensional function spaces. This has the inherent disadvantage that 16 variations in the not measured material properties are unknown. Therefore, an efficiency gain by 17 reason of the improvement of one, or more, of the material properties can be completely or partially 18 nullified by the worsening of other, not controlled, properties. This situation should be better 19 controlled in future cell manufacturing.

20 The benefits of mathematical modeling of the PSC's efficiency, by use of an analytical model

21 (Kratzenberg et al., 2015; Sun et al., 2015; Taretto et al., 2017), a numerical model (Amu, 2014; Azri et al., 2019; Devi et al., 2018; Dixit et al., 2019; Foster et al., 2014; Iftiquar and Yi, 2016; Kanoun et

23 al., 2019; Liu et al., 2014; Ren et al., 2017; Zhao et al., 2018), or a combination of these models

24 (Agarwal and Nair, 2014, 2015), as used to interpret the cell's drift-diffusion equations, can result in valuable understanding, and accelerate potentially the cell's development process. Similar analytical solutions of the drift-diffusion model were earlier introduced for organic solar cells (Jiang et al., 
1 2015; Chowdhury and Alam, 2014). Finite-difference modeling schemes, which present a better

2 accuracy, because of its higher resolution, are likewise, used to solve these equations (Zandi and

3 Razaghi, 2019), or to simulate the cell's short circuit current density $\left(J_{s c}\right)$ increase by reason of light

4 trapping (Cai et al., 2015). Some authors also present an efficiency optimization in a one-

5 dimensional optimization of the cells charge conduction layer thickness (Kim and Ohkita, 2017;

6 Zhao et al., 2018). Simulation studies based on the drift-diffusion models lead already in high

7 efficiency values of 25\% for single-junction PSCs (Agarwal and Nair, 2014, 2015). The drift-

8 diffusion models simulate the cell's current-voltage curve typically as a function of multiple material

9 properties and the absorber layer thickness. Considering arbitrary material property improvements at

10 different improvement scales, the efficiency of this model can be increased by a multidimensional

11 optimization algorithm. Multidimensional optimizations are state-of-the-art to solve many

12 engineering problems in order to find, e.g. (i) the optimal power flow in electric power grids (Crow,

13 2009), (ii) the lowest cost design of a wind turbine synchronous generator (Bazzo et al., 2017), or the

14 minimal series resistance in a GaAs solar cell (Algora and Díaz, 2000).

15 In the analyses of a cell's drift-diffusion model, a multidimensional optimization is useful in order to

16 demonstrate that the concomitant variable improvement results in much higher efficiency increases

17 than in state-of-the-art one- and two-dimensional optimization techniques. Additionally, the multidimensional optimization can lead (i) to a better understanding of the optimization process and

19 (ii) to the proposal of advanced solutions for future high-efficient solar cell design concepts as here

20 proposed.

21 While the highest state-of-the-art PCE of a manufactured PSC is already $24.2 \%$ (NREL, 2019),

22 perovskite solar cells show still a high improvement potential. Considering in a simplified PSC

23 model, only optical recombination losses, the maximal efficiencies of 30\% (Yin et al., 2014), 30.88\%

24 (Sha et al., 2015) and 31.3\% (Martynov et al., 2017) can theoretically be obtained in single-junction

25 PSCs. Furthermore, using zero surface recombination velocities and ideal light trapping in a drift-

26 diffusion model, a PCE of $29.9 \%$ can be obtained (Ren et al., 2017). These upper limits are below the 
1 highest attainable PCE of single-junction cells, which thermodynamic limit is calculated with $33.7 \%$

2 for the black-body (Shockley and Queisser, 1961), and 33\% for the measured irradiation spectrums 3 (Rühle, 2016).

4 However, in manufactured solar cells, additional recombination losses appear because of a cell's

5 non-ideal material properties. Based on the results from our multidimensional optimizations, we

6 propose cell designs that increase the efficiency of the state-of-the-art manufactured and simulated

7 PSCs. The same proposed cell designs present also a much lower Pb content. The high efficiency of 8 these cell designs is based on high-resolution $J_{s c}$ simulations of efficient light trapping schemes, and

9 we show how the PCE can be increased further, by additional tuning of the cell's material properties.

10 Similar design concepts can be found presently only for manufactured solar cells with much lower

11 PCE, in comparison to state-of-the-art cells (Liu, 2017), or otherwise, only for simulations of the $J_{s c}$

12 values (Cai et al., 2015). The former author shows that the efficiency of a manufactured PSC, with a

$13100 \mathrm{~nm}$ thick absorber layer, can increase from $5.8 \%$ to $7.1 \%$ by light trapping; and the latter authors

14 present a finite-difference time-domain simulation (FDTD), which shows that plasmonic light

15 trapping can lead in a 2.15-fold increase of the $J_{s c}$ value, in PSC with a $50 \mathrm{~nm}$ thin absorber layer, if 16 compared to a cell of this thickness without light trapping.

\section{Methodology - Optimization in a hypercube space of variables}

19 To develop a PSC, which shows the highest possible PCE, some fundamental questions should be answered. These are: (i) which multiple material properties and cell design parameters are necessary

21 to be improved concomitantly, (ii) has the value for each of the considered variables to be increased or decreased, and (iii) to which value each of these model variables should be ideally tuned. Using a

23 drift-diffusion model, these questions can be answered by searching the maximal efficiency in a multidimensional function space of material properties and manufacturing parameters. 
1 of this model are discussed in Appendix B1.1. The selected model and method reduce significantly

2 the computational optimization cost if compared to a numerical PSC model and random search methods. This enabled us to accomplish a large set of optimizations, using different constraints in

4 each of these optimizations, for the model's variable set. The approximations made by the selected

5 analytical model results in a low fitting deviation of only $0.1 \%$, between the measured and the

6 simulated PCE values (Sun et al., 2015). This small deviation is insignificant considering that

7 outdoor spectral variations can lead to PCE uncertainties of 3\% in thin-film cells (Rüther et al., 2002). The used model was elaborated in from (i) the second-order Poisson differential equation and its two boundary equations and (ii) a further set of four second-order drift-diffusion differential equations, and its boundary equations, which describe the cell's electron and hole transports in the dark and under exposition to light (Sun et al., 2015).

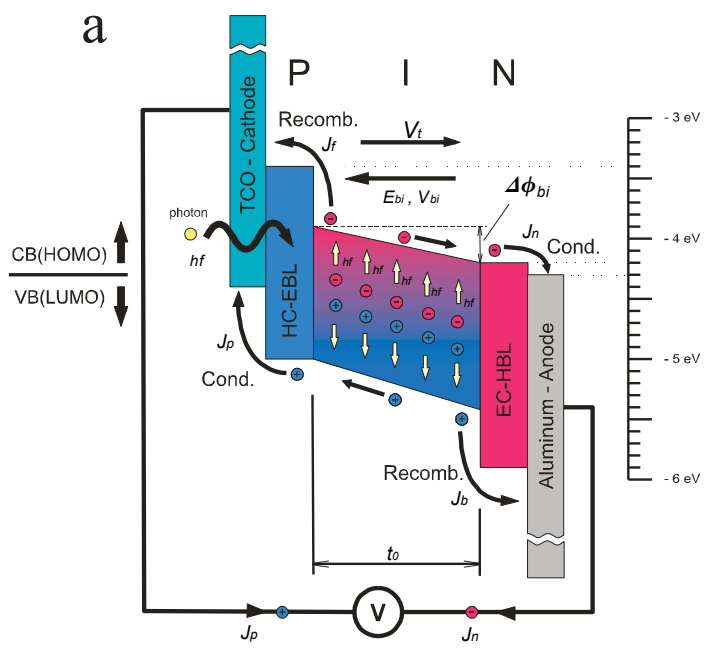

b

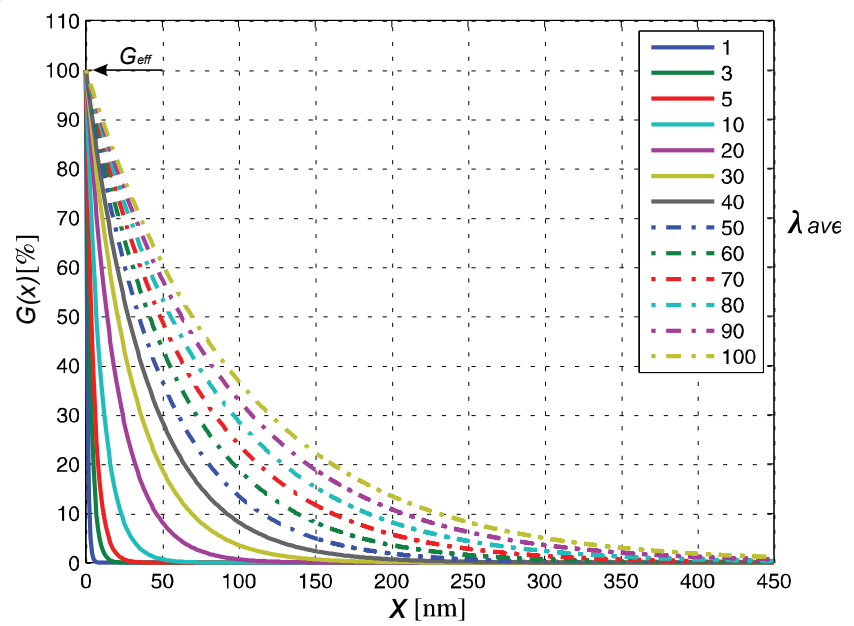

Fig. 1. Energy band diagram and normalized charge generation: (a) Energy potential diagram and charge carrier trajectories of a planar pin-type heterojunction perovskite solar cell, as modeled in (Sun et al. 2015), with the following components from left to right: (i) transparent top cover (here not visualized); (ii) cathode layer made of a Transparent Conductive Oxide (TCO); (iii) p-type hole conduction and electron blocking layer, made of organic material PEDOT:PSS; (iv) i-type intrinsic charge generation layer with thickness $t_{0}=450 \mathrm{~nm}$ made of the hybrid perovskite material; (v) n-type electron conduction and hole blocking layer, made of organic material PCBM; (vi) anode layer made of aluminum; adapted from supplementary information in (Sun et al. 2015). (b) Approximation of the normalized charge generation profiles $G(x)\left[\mathrm{cm}^{-2} \mathrm{~s}^{-1}\right]$ per solar cell area and time as a function of the penetration depth $(x=$ $0 \ldots 450 \mathrm{~nm}$ ) on the abscissa, calculated for several of the considered average optical decay lengths of $\lambda_{\text {ave }}=5$ to $150 \mathrm{~nm}$. FDTD simulations (Cai et al., 2015), our optimized solar cell architectures present light trapping 
1 nanoparticles in its thin absorber layers, which increase its short circuit current density $\left(J_{s c}\right)$, if

2 compared to the $J_{s c}=23 \mathrm{~mA} / \mathrm{cm}^{2}$ without light trapping, in a large range of absorber layer

3 thicknesses.

$4 \quad 3.1$ Modeling of the optimization

5 In this work, we propose the numerical optimization of the efficiency of PSC's analytical model in a

6 multidimensional function space, which is specified by the following optimization problem

$$
\eta_{\max } \rightarrow \max \left[\eta_{i}\left(X_{1, i} \ldots X_{9, i}\right)\right] \quad ; \quad i=1 \ldots N
$$

8 where the efficiency $\eta_{i}$ in the $i$-th maximization iteration is a function of the nine PSC model

9 variables $\left(X_{1, i \ldots} \ldots X_{9, i}\right)$, which built-up a nine-dimensional hypercube space, and where the maximum

10 efficiency $\left(\eta_{\max }\right)$ is obtained after $i=1 \ldots N$ model simulations. In a single iteration of this

11 optimization, each of the nine model variables is set up to a different value, and therefore, each

12 iteration results in a simulation $(i)$, where the variable values are selected by the optimization

13 algorithm. As initial model variables $\left(X_{1} \ldots X_{9}\right)$ we consider the values as obtained by the optimization

14 in (Sun et al., 2015). In each of the $N$ steps, the Matlab ${ }^{\mathrm{TM}}$ optimization algorithm fmincon improves

15 the values of the whole set of model variables by a Nonlinear Programming (NP) optimization

16 technique (Byrd et al., 1999), which is based on an interior point (IPM) and line search optimization

17 methods (LSM), in order to obtain the maximal possible model efficiency. When the cell's

18 efficiency increase is below a specified threshold value, the optimization algorithm considers that the

19 maximal cell efficiency is obtained, and it stops the optimization process (Fig. A.2). For each of the $i$

$20=1 \ldots N$ model simulations, a new calculation of (i) the J-V curves, (ii) the maximum power point

21 (MPP) power, and (iii) the cell's efficiency $\left(\eta_{i}\right)$ are accomplished, using equation (2). This PCE is

22 calculated as a function of nine model variables $X_{1, i}$ to $X 9, i$, which are constituted by eight quantum

23 physical material properties and the absorber layer thickness.

$$
\eta_{M P P, i}=\left(U_{M P P, i} J_{M P P, i}\left(X_{1, i} \ldots X_{9, i}\right)\right) / G_{A M 1.5}=P_{M P P, i} / G_{A M 1.5}
$$


2 In equation (2) $G_{A M 1.5}=100 \mathrm{~mW} / \mathrm{cm}^{2}$ is the normalized solar irradiation at Air Mass 1.5. The PSC's maximum output power density $P_{M P P, i}\left[\mathrm{~mW} / \mathrm{m}^{2}\right]$ of the $i$-th optimization step is obtained by the maximization of its power curve in $k=1$..M iterative steps as follows

$$
P_{M P P, i} \rightarrow \max \left(P_{k}\left(J_{\text {light, }}\left(G_{A M 1.5}, V_{k}, X_{1, i} \ldots X_{9, i}\right) \ldots J_{\text {light,M }}\left(G_{A M 1.5}, V_{k}\right)\right) ; k=1 \ldots M\right.
$$

where each $P_{M P P, i}$ is related to PSC's material properties in the $i$-th optimization step. In order to calculate $P_{M P P, i}$ the cell's analytical model uses the values of the whole set of model variables $\left(X_{1, i \ldots} \ldots X_{9, i}\right)$ as well as the terminal voltage $\left(V_{k}\right)$ and the cell's temperature as input variables to calculate its current density $J_{\text {light, } k}$ under exposure to reference light $\left(G_{A M 1.5}\right)$. The material properties are not modified in this second optimization. We remark that the power optimization of the equation (3) is nested in the efficiency optimization (equation 1). This nested optimization process is subject to the following specific boundary conditions

$$
X_{j, \text { min }} \leq X_{j} \leq X_{j, \max } \quad ; j=1 \ldots 9,
$$

where $X_{j, \max }$ and $X_{j, \min }$ are the maximal and minimal constraints for each one of the nine model variables $X_{j}$ to be optimized. Considering a large number of optimizations, each under different boundary conditions, specific constraints of the model variables in an arbitrary optimization process are given by equation (5). Each single optimization process considers a variable specific range $\left(X_{j-m i n}\right.$ $\left.\ldots X_{j, \max }\right)$, in which it is allowed for the optimization algorithm to modify each of the $j=1 \ldots 9$ model variables. Here we only consider for the built-in voltage $\left(V_{b i}\right)$, the absorber layer thickness $\left(t_{0}\right)$, and the average optical decay length ( $\left.\lambda_{\text {ave }}\right)$, variable specific constrains, as defined by the equations (6), (7) and (9). As we cannot know which property improvements will be possible in the future cell development, we set up arbitrary defined and adjustable constraints for remaining material properties, by a proposed variable boundary expansion factor $\left(f_{B}\right)$, as given by the following equation

$$
X_{j-\text { min }}=\left(1 / f_{B}\right) X_{j, m e} \leq X_{j} \leq X_{j, m e}\left(f_{B}\right)=X_{j, \text { max }} \quad ; \quad \mathrm{j}=1 \ldots 9,
$$


1 where the terms $f_{B}$ and $1 / f_{B}$ specify individual amplification and reduction factors in a single

2 optimization process. The application of $f_{B}$ results in the values of the upper and lower boundary

3 limits of the model variables $\left(X_{j-\min }\right.$ and $\left.X_{j \min }\right)$ in a specific efficiency optimization. In equation (5)

$4 X_{j, m e}$ represents the not expanded setup configuration of the model variables, as measured in (Sun et

5 al., 2015), and $X_{j-m i n}$ and $X_{j-m a x}$ are the minima and the maxima limits as specified in equation (4).

6 Built-in voltages with values $\left(V_{b i}>1.4 \mathrm{~V}\right)$ do not lead to any further increase of the cell's efficiency

7 (Fig. 2(b)). Hence, the inequality constraint equation (6) is imposed additionally to the optimization in order to avoid unrealistic high values of this variable. In fact, $f_{B}$ is used in this case to increase in small steps $V_{b i}$ to the maximal value of $X_{\max }=1.4 \mathrm{~V}$ (Tables 1 to 3 ).

$$
0 \leq V_{b i} \leq 1.4 \mathrm{~V}
$$

12 Furthermore, we consider that each of the different available coating techniques of the absorber layer 13 needs a specific minimal thickness $\left(t_{0-\min }\right)$ in order to avoid losses related to pinholes (Qiu et al., 14 2016) and conduction effects (Atwater and Polman, 2010). Therefore, we set up an additional constraint for $t$, which considers (i) an adjustable minimum thickness (t0-min) as the lower boundary in an optimization process, while (ii) the upper limit of $t_{0}$ is here assumed to be $1 \mu \mathrm{m}$ as specified by the inequality constraint equation (7).

In the Beer-Lambert law $\lambda_{\text {ave }}$ stands in a defined relationship with $t_{0}$, for the consideration of low reflection losses of approximately 1\% (Fig. 1b). This relation is expressed by equation ( 8 ) for the case 21 without light trapping (Sun et al., 2015).

$$
m=t_{0} / \lambda_{\text {ave }}
$$

23 With the setup values for $t_{0}$ and $\lambda_{\text {ave }}$ (section 3.2) the value of $m=4.5$, is obtained, which should also be considered for cells with light trapping, considering similar losses. Light trapping reduces the average optical decay length ( $\lambda_{\text {ave }}$ ) and improves at the same time the $J_{s c}$ (Cai et al., 2015). We use the calculated $m=4.5$ in order to specify an additional equality constraint (equation 9), here defined as the 
$\mathrm{m}$-constrained determination of $\lambda_{\text {ave. }}$ In this context we calculate $\lambda_{\text {ave }}\left(t_{0}\right)$ for light trapping as a function

4 If $\lambda_{\text {ave }}$ is instead in the same form constrained as the further model variables, by use of the $f_{B}$ factor, a 5 relationship appears, as defined by the vertex lines in Figs. $2 \mathrm{e}$ and $2 \mathrm{f}$, and Table A.3.2, which results

6 in lower $\lambda$ ave but higher optimized efficiency values (Tables 1 to 3 - last two columns). However, 7 improved absorption properties, and therefore, a reduced $\lambda_{\text {ave, }}$ as obtained by light trapping, should 8 stand in a similar relationship to the absorber layer thickness as in a cell without light trapping, by 9 reason of the Beer-Lambert law (Fig. 1b). Therefore, we calculate an $m$ - constrained $\lambda_{\text {ave }}$ in equation

10 (9), which results in a lower PCE, and presents, therefore, a more conservative formulation. In our $m$ 11 - constraint optimizations the remaining material properties are constrained by $f_{B}$ - factor and equations 12 (6) and (7). Whereas higher efficient light trapping schemes by shape-optimized plasmonic 13 nanoparticles are possible (Kakavelakis et al., 2017), the best light-trapping scheme, as here adopted, of $t_{0}$, solving equation (8) for $\lambda_{\text {ave }}$ as follows

$$
\lambda_{\text {ave }}\left(t_{0}\right)=t_{0} / m=t_{0} / 4.5
$$


1 We used the solar cell properties and $t_{0}$ as obtained in (Sun et al., 2015) as principal setup conditions.

2 The authors calculated by use of the OTM method, the fundamental optical parameters, which are: (i)

3 the effective generation of charge carriers, $G_{\text {eff }}=1.4356 \times 10^{13} \mathrm{~cm}^{-3} \mathrm{~s}^{-1}$, and (ii) the average optical

4 decay length $\lambda_{\text {ave }}=100 \mathrm{~nm}$, which are both specific material properties that are independent of absorber

5 layer thickness. By the derived equation for the total generation of free charge carriers (equations A.1

6 and A.2) a $G_{\max }$ of $1.4356 \times 10^{17} \mathrm{~cm}^{-2} \mathrm{~s}^{-1}$ is calculated, which coincides with the measured short circuit

7 current density of $q G_{\max }=23 \mathrm{~mA} / \mathrm{cm}^{2}$, where $q$ is the electric charge energy. Built-in voltage and the

8 diffusion coefficients are $V_{b i}=0.78 \mathrm{~V}$, and $D_{n}=D_{p}=0.05 \mathrm{~cm}^{2} / \mathrm{s}$. The authors obtained an ideal $t_{0}$ of

$9450 \mathrm{~nm}$ using a one-dimensional optimization of this variable. After the manufacturing of the PSC

10 with the optimized absorber layer thickness, the authors obtained by a curve fitting: (i) the surface

11 recombination velocity of electrons in the front charge transport layer $s_{f}=s_{n}=200 \mathrm{~cm} / \mathrm{s}$, (ii) the surface

12 recombination velocity of holes in the back charge transport layer $s_{p}=s b=19.2 \mathrm{~cm} / \mathrm{s}$, and (iii) the

13 number of the excess minority carrier concentrations of electrons and holes $\left(\Delta n=8.426 \times 10^{6} \mathrm{~cm}^{-3}, \Delta p\right.$

$\left.14=1.3003 \times 10^{8} \mathrm{~cm}^{-3}\right)$. Furthermore, they obtained an optimized PCE of $15.7 \%$, in a cell with an open-

15 circuit voltage of $V_{O C}=0.87 \mathrm{~V}$ (Table 4 - design 6$)$. The measured semiconductor temperature was

$16300.56 \mathrm{~K}$, which results in a thermal voltage of $V_{t}=k_{B} T / q=25.9 \mathrm{mV}$. As short circuit current densities

$17 q G_{\max }$ we adopted the values as obtained by the FDTD simulations in (Cai et al., 2015) for light

18 trapping with spherical nanoparticles, as a function of the absorber layer thickness of $t_{0}=50 \ldots 400 \mathrm{~nm}$

19 (equation 10)

\section{Results}

22 We propose (i) a multidimensional optimization of a solar cell's efficiency, as based on its drift23 diffusion model, and present additionally, (ii) a complete set of the accomplishable one- and two24 dimensional sensitivity analyses, as obtained by the possible variable combinations. Based on our results, we propose different cell designs, which increase the efficiency of state-of-the-art simulated 
1 and manufactured cells. Figs. 2(a) to 2(e) show some typical one- and two-dimensional, sensitivity

2 analyses, where the efficiency is calculated as a function of the improvement of its model variables. 3 


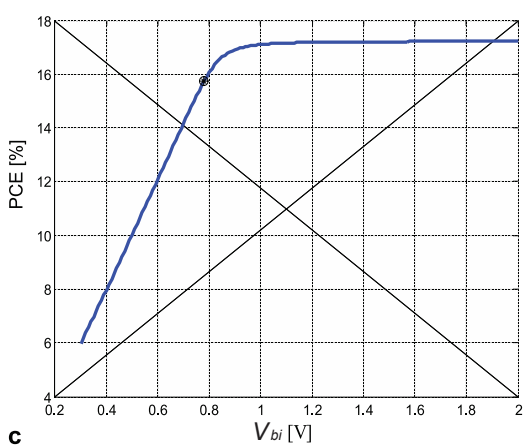

$$
\text { c }
$$
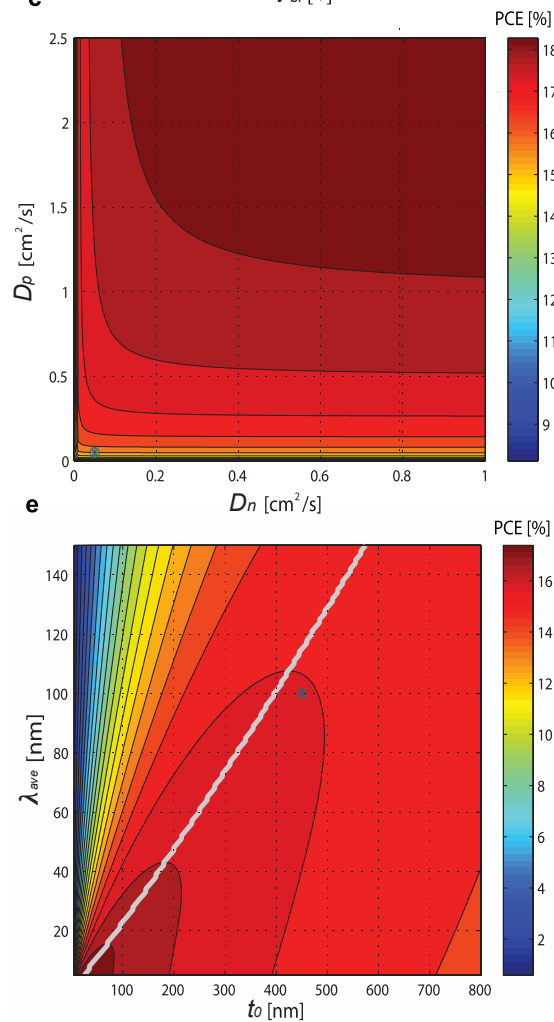

b

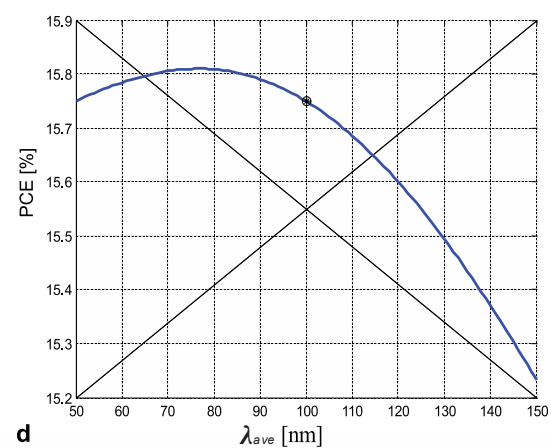

d $\times 10^{9}$
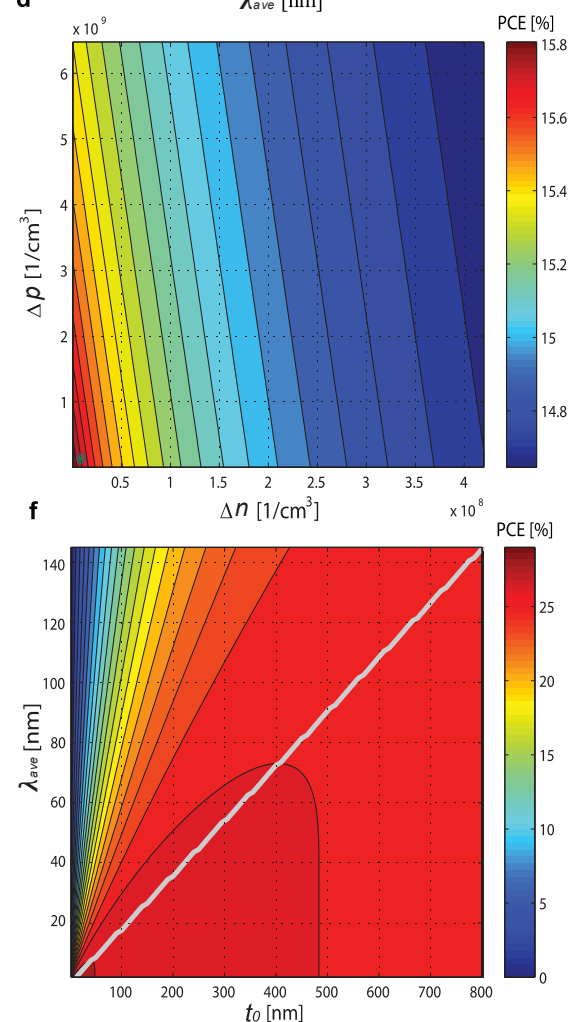

Fig. 2. One- and two-dimensional sensitivity analyses, showing the model efficiency as a function of: (a) the average optical decay length $\lambda_{a v e}$; (b) the built-in voltage $V_{b i}$; (c) the diffusion coefficients of electrons and holes $D_{n}$ and $D_{p}$; (d) the excess concentration of electrons and holes $\Delta n$ and $\Delta p$; and finally, in (e) and (f), the absorber layer thickness $t$, and the average optical decay length, where the grey vertex line shows the maximal attainable efficiency values in these two-dimensional presentations; (a) - (e) the remaining model variables are set up to the values as obtained in (Sun et al. 2015); (f) the values of the remaining model variables are set up to the values as obtained in our $f_{B}$ constrained multidimensional optimization for $f_{B}=160$; the measured efficiency values of $15.7 \%$ in (a) - (e) appear as a star within a surrounded circle.

For the visualization of the efficiency gains, as obtained by the possible combinations of nine model variables, a large set of 45 such figures would be necessary. However, these figures can be substituted by Table A.1, which shows only the highest attainable PCE values of those analyses, as a function of different boundary expansion factors $f_{B}$ (equation 5). For the highest variable improvement factor $\left(f_{B}=160\right)$ the best one- and two-dimensional efficiency optimizations increase the PCE from $15.7 \%$ to $18.1 \%$ and $20 \%$. The Figs. 2(e) and 2(f) compare two similar two- 
1 dimensional sensitivity analyses using the same model variables. While in Fig. 2(e) the remaining

2 variables confer to our setup condition, in Fig. 2(f) these variables are configured with the ideal

3 values as obtained by a multidimensional optimization with $f_{B}=160$. Both figures consider light

4 trapping with independently adjustable $t_{0}$ and $\lambda_{\text {ave, }}$, but the multidimensional optimization results in

5 higher efficiency values. It can be observed from the slight shift of the grey vertex lines in these

6 figures that for arbitrary values of $t$, the multidimensional optimization demands an improved light

7 trapping, by reason of the lower optimal values of $\lambda_{\text {ave }}$ for the same $t$, , if compared to the two-

8 dimensional optimization. Based on the results of our $f_{B}$ - constraint multidimensional optimization,

9 as presented in Table A.2, we conclude that a variable relationship that results in maximal efficiency

10 vertex lines does only appear in-between the variables $t_{0}$ and $\lambda_{\text {ave, }}$ and not in-between the further

11 model variables.

12 Fig. 3 presents six sets of 160 multidimensional optimizations $\left(f_{B}=1 \ldots 160\right)$, for six different

13 absorber layer thicknesses. For these $m$-constrained optimizations the variables $V_{b i}, t o, \lambda_{a v e}$ and the

14 short circuit current densities are set up by the equations (6), (7), (9) and (10), while the remaining

15 variables are $f_{B}$ - constrained. As specified in equation (7), we consider that each coating technique of

16 the absorber has its own inherent minimum thickness (to-min) for the deposition of perfect absorber

17 layers (Appendix section A.4), and therefore, we present optimized designs for different absorber

18 layer thicknesses. Light trapping with spherical nanoparticles (solid curves) presents a significant

19 efficiency advantage in comparison to the PSC without light trapping (dotted curve) in almost all

20 development states that are expressed by the $f_{B}$ factor. In a $160 \mathrm{~nm}$ thick absorber layer, such a light-

21 trapping shows only $0.1 \ldots 0.2 \%$ lower efficiencies, in comparison to the configuration with the

22 highest efficiencies $\left(t_{0}=400 \mathrm{~nm}\right)$. Additionally, a PSC with $t_{0}=160$ has the advantage of presenting

23 2.5-fold lower $\mathrm{Pb}$ content, and therefore, we give special emphasis to this cell design in our

24 discussions. 


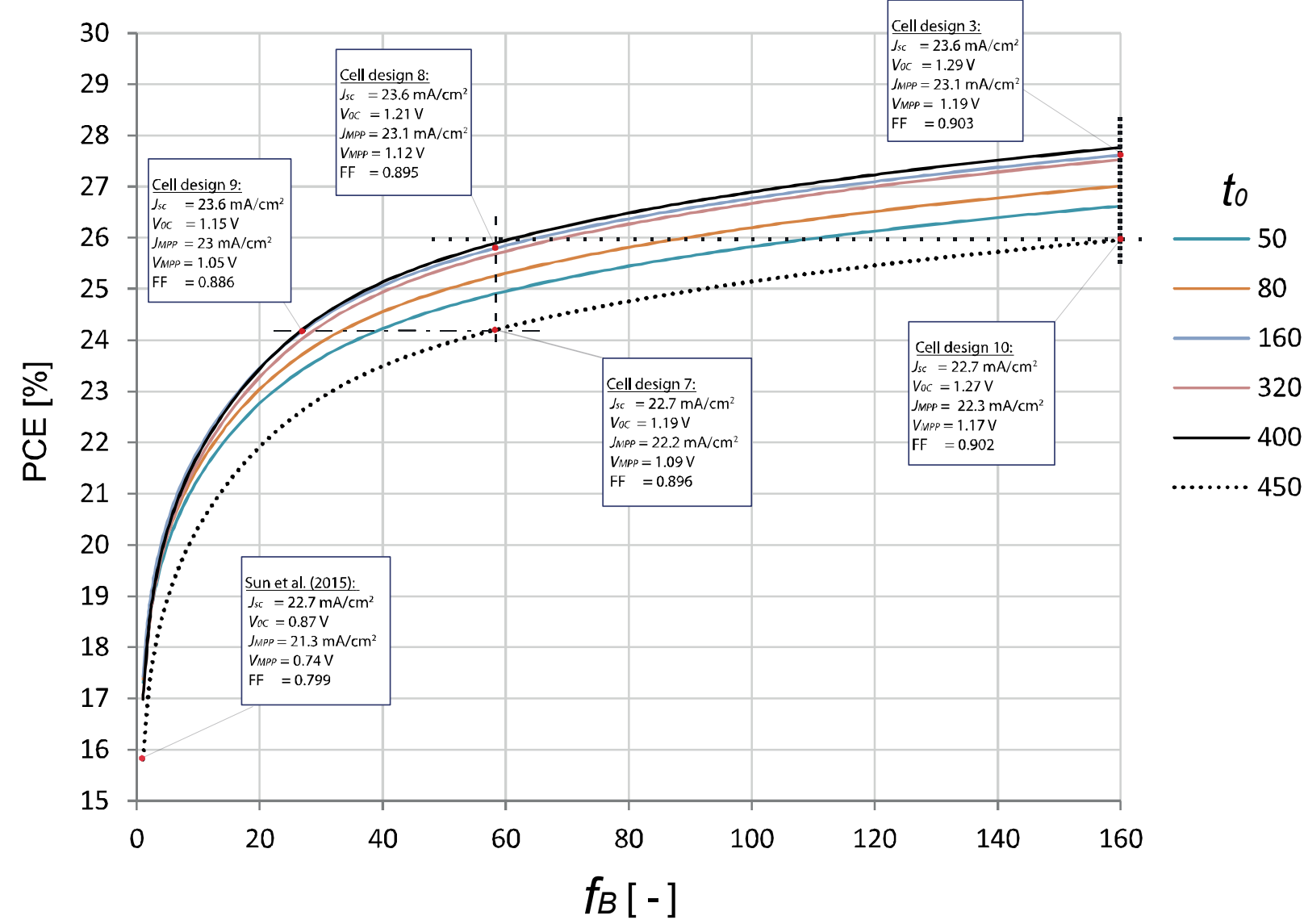

Fig. 3. Optimized efficiency as a function of the 160 boundary expansion factors $\left(f_{B}=1 \ldots 160, \in \mathbb{N}\right)$ for six absorber layer thicknesses in the range of $t_{0}=50 \ldots 450 \mathrm{~nm}$, optimization variables $\left(D_{n}, D_{p}, V_{b i}, s_{f}, s_{b}\right)$, while $\lambda_{\text {ave }}\left(t_{0}\right)=t_{0} / 4.5$ is the calculated average optical decay length: (i) without light trapping, $t_{0-\min }=450 \mathrm{~nm}$, $\lambda_{\text {ave }}=100 \mathrm{~nm}$; (ii) light trapping with spherical plasmonic nanoparticles, $t_{0}=$ $50 \ldots . .400 \mathrm{~nm}$, with corrected short circuit current densities. Used $q G_{\max }$ values, in unit $\left[\mathrm{mA} / \mathrm{cm}^{2}\right]$, as obtained in (Cai et al. 2015 and Sun et al. 2015): $22.5\left(t_{0}=50 \mathrm{~nm}\right), 23.05(80 \mathrm{~nm}), 23.9(160 \mathrm{~nm}), 24.18(320 \mathrm{~nm}), 24.5(400 \mathrm{~nm}), 23(450 \mathrm{~nm}$, without light trapping).

In Tables 1, 2 and 3, we show an alternative representation for some optimizations in Fig. 3, which also show the values of the optimized material properties. While Table 1 considers the state-of-theart absorber layer thickness of $450 \mathrm{~nm}$ and no light trapping, Tables 2 and 3 present the results for thinner absorber layers of $400 \mathrm{~nm}$ and $160 \mathrm{~nm}$, considering a $J_{s c}$ as obtained by light trapping with spherical nanoparticles and a corrected $\lambda_{\text {ave }}$ by equation (9). For the interested reader, we additionally show the PCE and $\lambda_{\text {ave }}$ values, as obtained in optimizations with $f_{B}$ - constrained $\lambda_{\text {ave }}$ setups (equation 5). However, these values should be observed with caution, because of the related low reflection losses, which might be unrealistic. Figs. $4 \mathrm{a}$ and $4 \mathrm{~b}$ present, the J-V curves, and the power curves, of nine different high-efficiency design proposals. The figure visualizes in more detail, some of the simulated design proposals as 
1 presented in Fig. 3. Highest efficiency designs (1) to (5) show light trapping for $t_{0}=50 \ldots 400 \mathrm{~nm}$,

2 with $f_{B}=160$. While design (6) presents the cell as proposed is (Sun et al., 2015), (7) confers, as an

3 example, to the cell with the state-of-the-art efficiency, considering improved material properties and

4 no light trapping. Design (8) shows how (7) can be improved by light trapping, and (9) shows a

5 similar efficiency as (7), with less ideal properties, but with light trapping. Table 4 presents the J-V

6 performance parameters of the design proposals as shown in Figs. 3 and 4, and Table 5 visualizes the

7 relative improvements, considering in each row the comparison of two of the design proposals, as

8 presented in Table 4.

Table 1

Optimized model variables (boldface symbols) as obtained from optimizations in a seven-dimensional function space considering eleven optimizations with specific boundary expansion factors $f_{B}$, obtaining an ideal PSC of $25.96 \%$, for a cell with a fixed $t_{0}$ of $450 \mathrm{~nm}$, which do not use light trapping $\left(\lambda_{\text {ave }}=100 \mathrm{~nm}\right) ; q G_{\max }=23 \mathrm{~mA} / \mathrm{cm}^{2}$.

\begin{tabular}{rrrrrrrrrrrrrrr}
\hline$f_{B}$ & $\begin{array}{r}\boldsymbol{s}_{f} \\
{[\mathrm{c} / \mathrm{s}]}\end{array}$ & $\begin{array}{r}\boldsymbol{s}_{\boldsymbol{b}} \\
{[\mathrm{cm} / \mathrm{s}]}\end{array}$ & $\begin{array}{r}\Delta \boldsymbol{n} \\
{\left[1 / \mathrm{cm}^{3}\right]}\end{array}$ & $\begin{array}{r}\Delta \boldsymbol{p} \\
{\left[1 / \mathrm{cm}^{3}\right]}\end{array}$ & $\begin{array}{r}\boldsymbol{V}_{\boldsymbol{b i}} \\
{[\mathrm{V}]}\end{array}$ & $\begin{array}{r}\boldsymbol{D}_{\boldsymbol{n}} \\
{\left[\mathrm{cm}^{2} / \mathrm{s}\right]}\end{array}$ & $\begin{array}{r}\boldsymbol{D}_{\boldsymbol{p}} \\
{\left[\mathrm{cm}^{2} / \mathrm{s}\right]}\end{array}$ & $\begin{array}{r}\mu_{n} \\
{\left[\mathrm{~cm}^{2} / \mathrm{Vs}\right]}\end{array}$ & $\begin{array}{r}\mu_{p} \\
{\left[\mathrm{~cm}^{2} / \mathrm{Vs}\right]}\end{array}$ & $\begin{array}{r}t_{0} \\
{[\mathrm{~nm}]}\end{array}$ & $\begin{array}{r}\lambda_{\text {ave }} \\
{[\mathrm{nm}]}\end{array}$ & $\begin{array}{r}\eta \\
{[\%]}\end{array}$ & $\begin{array}{r}\lambda_{a v e}{ }^{*} \\
{[\mathrm{~nm}]}\end{array}$ & $\begin{array}{r}\eta^{*} \\
{[\%]}\end{array}$ \\
\hline 5.00 & 40.00 & 3.84 & $8.43 \mathrm{E}+06$ & $1.30 \mathrm{E}+08$ & 0.80 & 0.25 & 0.25 & 9.65 & 9.65 & 450.00 & 100.00 & 18.96 & 48.13 & 19.15 \\
10.00 & 20.00 & 1.92 & $8.43 \mathrm{E}+06$ & $1.30 \mathrm{E}+08$ & 0.82 & 0.50 & 0.50 & 19.30 & 19.30 & 450.00 & 100.00 & 20.34 & 10.00 & 20.57 \\
20.00 & 10.00 & 0.96 & $8.43 \mathrm{E}+06$ & $1.30 \mathrm{E}+08$ & 0.86 & 1.00 & 1.00 & 38.61 & 38.61 & 450.00 & 100.00 & 21.92 & 5.00 & 22.17 \\
30.00 & 6.67 & 0.64 & $8.43 \mathrm{E}+06$ & $1.30 \mathrm{E}+08$ & 0.90 & 1.50 & 1.50 & 57.91 & 57.91 & 450.00 & 100.00 & 22.88 & 3.33 & 23.14 \\
40.00 & 5.00 & 0.48 & $8.43 \mathrm{E}+06$ & $1.30 \mathrm{E}+08$ & 0.94 & 2.00 & 2.00 & 77.22 & 77.22 & 450.00 & 100.00 & 23.49 & 2.50 & 23.76 \\
60.00 & 3.33 & 0.32 & $8.43 \mathrm{E}+06$ & $1.30 \mathrm{E}+08$ & 1.01 & 3.00 & 3.00 & 115.82 & 115.82 & 450.00 & 100.00 & 24.26 & 1.67 & 24.53 \\
80.00 & 2.50 & 0.24 & $8.43 \mathrm{E}+06$ & $1.30 \mathrm{E}+08$ & 1.09 & 4.00 & 4.00 & 154.43 & 154.43 & 450.00 & 100.00 & 24.76 & 27.89 & 25.04 \\
100.00 & 2.00 & 0.19 & $8.43 \mathrm{E}+06$ & $1.30 \mathrm{E}+08$ & 1.17 & 5.00 & 5.00 & 193.04 & 193.04 & 450.00 & 100.00 & 25.14 & 26.85 & 25.43 \\
120.00 & 1.67 & 0.16 & $8.43 \mathrm{E}+06$ & $1.30 \mathrm{E}+08$ & 1.25 & 6.00 & 6.00 & 231.65 & 231.65 & 450.00 & 100.00 & 25.46 & 25.85 & 25.75 \\
140.00 & 1.43 & 0.14 & $8.43 \mathrm{E}+06$ & $1.30 \mathrm{E}+08$ & 1.32 & 7.00 & 7.00 & 270.26 & 270.26 & 450.00 & 100.00 & 25.73 & 25.01 & 26.02 \\
160.00 & 1.25 & 0.12 & $8.43 \mathrm{E}+06$ & $1.30 \mathrm{E}+08$ & 1.40 & 8.00 & 8.00 & 308.87 & 308.87 & 450.00 & 100.00 & 25.96 & 0.63 & 26.26 \\
\hline
\end{tabular}

Observations: The values in italic formatted numbers correspond to the values of the lower curve in Fig. 3; *values obtained in $f_{B}-$ constrained determination of $\lambda_{a v e}$

Table 2

Optimized model variables (boldface symbols) and efficiency in seven-dimensional optimizations as a function of several boundary extension factors $f_{B}$ for a PSC with $t_{0}=400 \mathrm{~nm}$, where light trapping by use of spherical nanoparticles compensates the reduced absorption in this absorber layer; $q G_{\max }=24.5 \mathrm{~mA} / \mathrm{cm}^{2}$.

\begin{tabular}{rrrrrrrrrrrrrrr}
\hline$f_{B}$ & $\boldsymbol{s}_{\boldsymbol{f}}$ & $\boldsymbol{s}_{\boldsymbol{b}}$ & $\begin{array}{r}\boldsymbol{\Delta n} \\
{[\mathrm{cm} / \mathrm{s}]}\end{array}$ & $\begin{array}{r}\boldsymbol{\Delta} \boldsymbol{p} \\
{\left[\mathrm{cm} / \mathrm{cm}^{3}\right]}\end{array}$ & $\begin{array}{r}\boldsymbol{V}_{\boldsymbol{b i}} \\
{\left[1 / \mathrm{cm}^{3}\right]}\end{array}$ & $\begin{array}{r}\boldsymbol{D}_{\boldsymbol{n}} \\
{[\mathrm{V}]}\end{array}$ & $\begin{array}{r}\boldsymbol{D}_{\boldsymbol{p}} \\
{\left[\mathrm{cm}^{2} / \mathrm{s}\right]}\end{array}$ & $\begin{array}{r}\mu_{n} \\
{\left[\mathrm{~cm}^{2} / \mathrm{s}\right]}\end{array}$ & $\begin{array}{r}\mu_{p} \\
{\left[\mathrm{~cm}^{2} / \mathrm{Vs}\right]}\end{array}$ & $\begin{array}{r}t_{0} \\
{\left[\mathrm{~cm}^{2} / \mathrm{Vs}\right]}\end{array}$ & $\begin{array}{r}\lambda_{\text {ave }} \\
{[\mathrm{nm}]}\end{array}$ & $\begin{array}{r}\eta \\
{[\mathrm{nm}]}\end{array}$ & $\begin{array}{r}\lambda_{\text {ave }}{ }^{*} \\
{[\mathrm{~nm}]}\end{array}$ & $\begin{array}{r}\eta^{*} \\
{[\%]}\end{array}$ \\
\hline 5.00 & 40.00 & 3.84 & $8.43 \mathrm{E}+06$ & $1.30 \mathrm{E}+08$ & 0.80 & 0.25 & 0.25 & 9.65 & 9.65 & 400.00 & 88.89 & 20.29 & 41.76 & 20.50 \\
10.00 & 20.00 & 1.92 & $8.43 \mathrm{E}+06$ & $1.30 \mathrm{E}+08$ & 0.82 & 0.50 & 0.50 & 19.30 & 19.30 & 400.00 & 88.89 & 21.76 & 8.89 & 22.01 \\
20.00 & 10.00 & 0.96 & $8.43 \mathrm{E}+06$ & $1.30 \mathrm{E}+08$ & 0.86 & 1.00 & 1.00 & 38.61 & 38.61 & 400.00 & 88.89 & 23.45 & 4.44 & 23.72 \\
30.00 & 6.67 & 0.64 & $8.43 \mathrm{E}+06$ & $1.30 \mathrm{E}+08$ & 0.90 & 1.50 & 1.50 & 57.91 & 57.91 & 400.00 & 88.89 & 24.47 & 2.96 & 24.76 \\
40.00 & 5.00 & 0.48 & $8.43 \mathrm{E}+06$ & $1.30 \mathrm{E}+08$ & 0.94 & 2.00 & 2.00 & 77.22 & 77.22 & 400.00 & 88.89 & 25.13 & 2.22 & 25.42 \\
60.00 & 3.33 & 0.32 & $8.43 \mathrm{E}+06$ & $1.30 \mathrm{E}+08$ & 1.01 & 3.00 & 3.00 & 115.82 & 115.82 & 400.00 & 88.89 & 25.95 & 1.48 & 26.25 \\
80.00 & 2.50 & 0.24 & $8.43 \mathrm{E}+06$ & $1.30 \mathrm{E}+08$ & 1.09 & 4.00 & 4.00 & 154.43 & 154.43 & 400.00 & 88.89 & 26.48 & 24.56 & 26.79 \\
100.00 & 2.00 & 0.19 & $8.43 \mathrm{E}+06$ & $1.30 \mathrm{E}+08$ & 1.17 & 5.00 & 5.00 & 193.04 & 193.04 & 400.00 & 88.89 & 26.90 & 23.71 & 27.17 \\
120.00 & 1.67 & 0.16 & $8.43 \mathrm{E}+06$ & $1.30 \mathrm{E}+08$ & 1.25 & 6.00 & 6.00 & 231.65 & 231.65 & 400.00 & 88.89 & 27.23 & 22.83 & 27.54 \\
140.00 & 1.43 & 0.14 & $8.43 \mathrm{E}+06$ & $1.30 \mathrm{E}+08$ & 1.32 & 7.00 & 7.00 & 270.26 & 270.26 & 400.00 & 88.89 & 27.52 & 22.15 & 27.83 \\
160.00 & 1.25 & 0.12 & $8.43 \mathrm{E}+06$ & $1.30 \mathrm{E}+08$ & 1.40 & 8.00 & 8.00 & 308.87 & 308.87 & 400.00 & 88.89 & 27.76 & 21.50 & 28.08 \\
\hline
\end{tabular}

Observations: The values in italic formatted numbers correspond to the cell with $t_{0}=400 \mathrm{~nm}$ in Fig. 3 ; * values obtained in $f_{B}-$ constrained determination of $\lambda_{\text {ave }}$ 


\section{Table 3}

Optimized model variables (boldface symbols) and efficiencies in seven-dimensional optimizations as a function of several boundary extension factors $f_{B}$ for a PSC with $t_{0}=160 \mathrm{~nm}$, where light trapping by spherical nanoparticles compensates the reduced absorption in this absorber layer; $q G_{\max }=23.9 \mathrm{~mA} / \mathrm{cm}^{2}$.

\begin{tabular}{|c|c|c|c|c|c|c|c|c|c|c|c|c|c|c|}
\hline $\begin{array}{l}f_{B} \\
{[-]}\end{array}$ & $\begin{array}{r}\boldsymbol{S}_{\boldsymbol{f}} \\
{[\mathrm{cm} / \mathrm{s}]}\end{array}$ & $\begin{array}{r}S_{b} \\
{[\mathrm{~cm} / \mathrm{s}]}\end{array}$ & $\begin{array}{r}\Delta \boldsymbol{n} \\
{\left[1 / \mathrm{cm}^{3}\right]}\end{array}$ & $\begin{array}{r}\Delta p \\
{\left[1 / \mathrm{cm}^{3}\right]}\end{array}$ & $\begin{array}{l}V_{b i} \\
{[\mathrm{~V}]}\end{array}$ & $\begin{array}{r}D_{n} \\
{\left[\mathrm{~cm}^{2} / \mathrm{s}\right]}\end{array}$ & $\begin{array}{r}\boldsymbol{D}_{\boldsymbol{p}} \\
{\left[\mathrm{cm}^{2} / \mathrm{s}\right]}\end{array}$ & $\begin{array}{r}\mu_{n} \\
{\left[\mathrm{~cm}^{2} / \mathrm{Vs}\right]}\end{array}$ & $\begin{array}{r}\mu_{p} \\
{\left[\mathrm{~cm}^{2} / \mathrm{Vs}\right]}\end{array}$ & $\begin{array}{r}t_{0} \\
{[\mathrm{~nm}]}\end{array}$ & $\begin{array}{r}\lambda_{a v e} \\
{[\mathrm{~nm}]}\end{array}$ & $\begin{array}{r}\eta \\
{[\%]}\end{array}$ & $\begin{array}{l}\lambda_{a v e}{ }^{*} \\
{[\mathrm{~nm}]}\end{array}$ & $\begin{array}{c}\eta^{*} \\
{[\%]}\end{array}$ \\
\hline 5.00 & 40.00 & 3.84 & $8.43 \mathrm{E}+06$ & $1.30 \mathrm{E}+08$ & 0.80 & 0.25 & 0.25 & 9.65 & 9.65 & 160.00 & 35.56 & 20.46 & 7.11 & 21.20 \\
\hline 10.00 & 20.00 & 1.92 & $8.43 \mathrm{E}+06$ & $1.30 \mathrm{E}+08$ & 0.82 & 0.50 & 0.50 & 19.30 & 19.30 & 160.00 & 35.56 & 21.85 & 3.56 & 22.67 \\
\hline 20.00 & 10.00 & 0.96 & $8.43 \mathrm{E}+06$ & $1.30 \mathrm{E}+08$ & 0.86 & 1.00 & 1.00 & 38.61 & 38.61 & 160.00 & 35.56 & 23.46 & 1.78 & 24.34 \\
\hline 30.00 & 6.67 & 0.64 & $8.43 \mathrm{E}+06$ & $1.30 \mathrm{E}+08$ & 0.90 & 1.50 & 1.50 & 57.91 & 57.91 & 160.00 & 35.56 & 24.43 & 1.19 & 25.35 \\
\hline 40.00 & 5.00 & 0.48 & $8.43 \mathrm{E}+06$ & $1.30 \mathrm{E}+08$ & 0.94 & 2.00 & 2.00 & 77.22 & 77.22 & 160.00 & 35.56 & 25.06 & 0.89 & 26.00 \\
\hline 60.00 & 3.33 & 0.32 & $8.43 \mathrm{E}+06$ & $1.30 \mathrm{E}+08$ & 1.01 & 3.00 & 3.00 & 115.82 & 115.82 & 160.00 & 35.56 & 25.84 & 0.59 & 26.82 \\
\hline 80.00 & 2.50 & 0.24 & $8.43 \mathrm{E}+06$ & $1.30 \mathrm{E}+08$ & 1.09 & 4.00 & 4.00 & 154.43 & 154.43 & 160.00 & 35.56 & 26.37 & 9.08 & 27.36 \\
\hline 100.00 & 2.00 & 0.19 & $8.43 \mathrm{E}+06$ & $1.30 \mathrm{E}+08$ & 1.17 & 5.00 & 5.00 & 193.04 & 193.04 & 160.00 & 35.56 & 26.77 & 0.60 & 27.77 \\
\hline 120.00 & 1.67 & 0.16 & $8.43 \mathrm{E}+06$ & $1.30 \mathrm{E}+08$ & 1.25 & 6.00 & 6.00 & 231.65 & 231.65 & 160.00 & 35.56 & 27.10 & 0.90 & 28.11 \\
\hline 140.00 & 1.43 & 0.14 & $8.43 \mathrm{E}+06$ & $1.30 \mathrm{E}+08$ & 1.32 & 7.00 & 7.00 & 270.26 & 270.26 & 160.00 & 35.56 & 27.37 & 8.44 & 28.40 \\
\hline 160.00 & 1.25 & 0.12 & $8.43 \mathrm{E}+06$ & $1.30 \mathrm{E}+08$ & 1.40 & 8.00 & 8.00 & 308.87 & 308.87 & 160.00 & 35.56 & 27.61 & 0.71 & 28.65 \\
\hline
\end{tabular}

Observations: (i) The values in boldface formatted numbers correspond to cell design 3 in Fig.4, and high-efficiency cell in Fig. 5; (ii) the values in italic formatted numbers correspond to the cell with $t_{0}=160 \mathrm{~nm}$ in Fig. 3; * values obtained in $f_{B}$ - constrained determination of $\lambda_{\text {ave. }}$

3 The curves in Fig. 3 visualize how the efficiency of a PSC in a certain development state can be

4 optimized by (i) the improvement of the material properties, as expressed by the $f_{B}$ factor; (ii) light

5 trapping for a constant $f_{B}$ factor, as used for $t_{0}<450 \mathrm{~nm}$; and (iii) the combination of these methods.

6 While material property improvements result in efficiency growths almost solely based on the

7 increase of $V_{M P P}$; light trapping increases the efficiency as a function of both, a $V_{M P P}$ rise, and

8 predominantly, an increase of $J_{M P P}$. Such improvements can be discussed comparing the optimization

9 results in Table 4 (Fig.4), which results in Table 5. Considering that the cell with the state-of-the-art

10 efficiency of $24.2 \%$ is based on an effective light trapping scheme (Figure 3, design 9), a relative

11 efficiency rise of $14.6 \%$ (Table 5 - line 13) can be obtained as a function of further material property

12 improvements. This increase is almost solely based on a $14.1 \%$ rise of $V_{M P P}$ and results in the

13 proposed cell design 3 . 

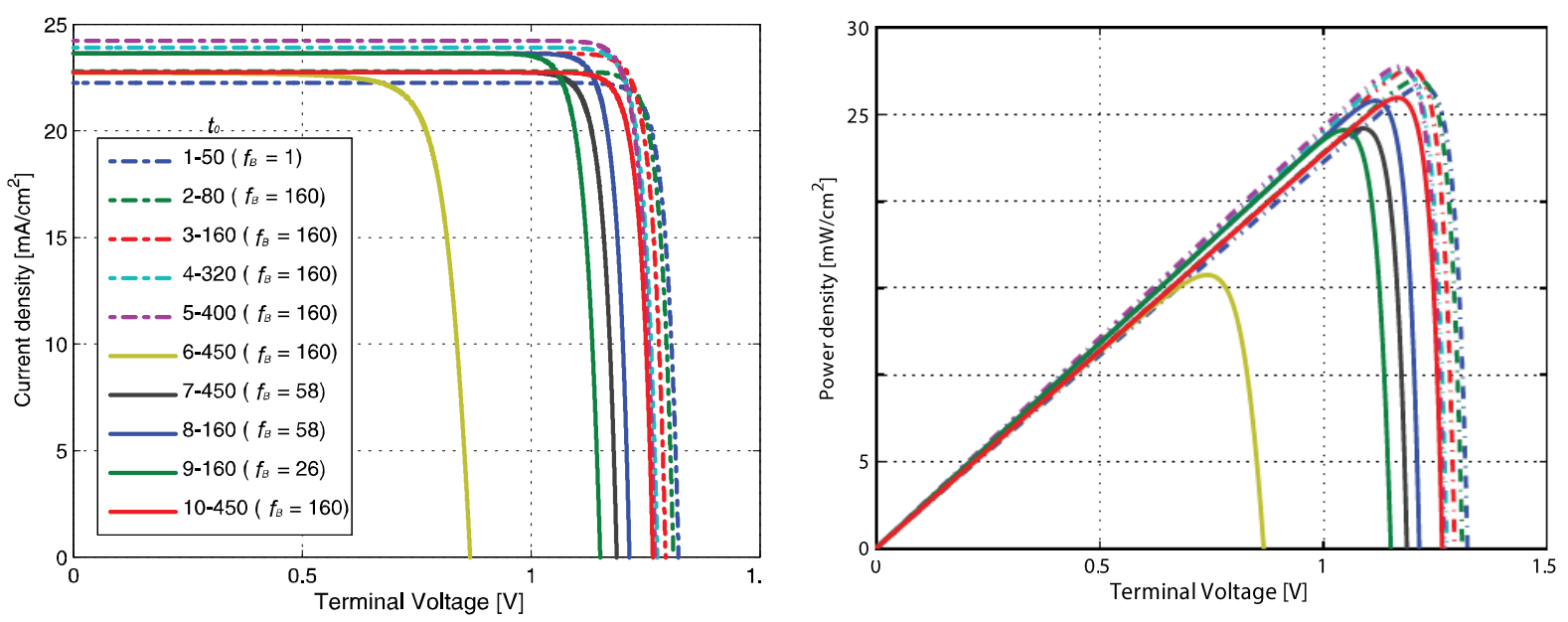

Fig. 4. (a) J-V and (b) power curves of different solar cell designs, as obtained by the multidimensional optimizations, as specified by the absorber layer thickness $\left(t_{0}\right)$ and boundary amplification factor $\left(f_{B}\right)$ : (1) $t_{0}=50 \mathrm{~nm}, f_{B}=160 ;(2) t_{0}=80 \mathrm{~nm}, f_{B}=160 ;(3) t_{0}=160$ $\mathrm{nm}, f_{B}=160$; (4) $t_{0}=320 \mathrm{~nm}, f_{B}=160 ;(5) t_{0}=400 \mathrm{~nm}, f_{B}=160$; (6) $t_{0}=450 \mathrm{~nm}, f_{B}=1$ (Sun et al., 2015); (7) $t_{0}=450 \mathrm{~nm}, f_{B}=58 \approx$ state-of-the-art efficiency value; (8) $t_{0}=160 \mathrm{~nm}, f_{B}=58$; (9) $t_{0}=160 \mathrm{~nm}, f_{B}=26 \approx$ state-of-the-art efficiency value, (10) $t_{0}=450, f_{B}=$ 160.

\section{7}

Table 4

Optimization setup configurations and perovskite performance parameters extracted from the J-V curve characteristics for the cell designs as presented in Figs. 3 and 4.

\begin{tabular}{lrrrrrrrrrr}
\hline $\begin{array}{l}\text { Cell } \\
\text { design }\end{array}$ & $\begin{array}{r}\boldsymbol{f}_{\boldsymbol{B}} \\
{[-]}\end{array}$ & $\begin{array}{r}\boldsymbol{t}_{\boldsymbol{o}} \\
{[\mathrm{nm}]}\end{array}$ & $\begin{array}{r}\lambda_{\text {ave }} \\
{[\mathrm{nm}]}\end{array}$ & $\begin{array}{r}\boldsymbol{q} \boldsymbol{G}_{\max }(\#) \\
{\left[\mathrm{mA} / \mathrm{cm}^{2}\right]}\end{array}$ & $\begin{array}{r}\boldsymbol{J}_{\boldsymbol{S C}} \\
{\left[\mathrm{mA} / \mathrm{cm}^{2}\right]}\end{array}$ & $\begin{array}{r}\boldsymbol{V}_{\boldsymbol{O C}} \\
{[\mathrm{V}]}\end{array}$ & $\begin{array}{r}\boldsymbol{J}_{\boldsymbol{M P P}} \\
{\left[\mathrm{mA} / \mathrm{cm}^{2}\right]}\end{array}$ & $\begin{array}{r}V_{M P P} \\
{[\mathrm{~V}]}\end{array}$ & $\begin{array}{r}\mathrm{FF} \\
{[-]}\end{array}$ & $\begin{array}{r}\text { PCE } \\
{[\%]}\end{array}$ \\
\hline $\mathbf{1}$ & 160 & 50 & 11.1 & 22.5 & 22.3 & 1.32 & 21.8 & 1.22 & 0.905 & 26.6 \\
$\mathbf{2}$ & 160 & 80 & 17.8 & 23.1 & 22.8 & 1.31 & 22.3 & 1.21 & 0.904 & 27.0 \\
$\mathbf{3}$ & 160 & 160 & 35.6 & 23.9 & 23.6 & 1.29 & 23.1 & 1.19 & 0.903 & 27.6 \\
$\mathbf{4}$ & 160 & 320 & 71.1 & 24.2 & 23.9 & 1.28 & 23.4 & 1.18 & 0.902 & 27.5 \\
$\mathbf{5}$ & 160 & 400 & 88.9 & 24.5 & 24.2 & 1.27 & 23.7 & 1.17 & 0.902 & 27.8 \\
$\mathbf{6}$ & 1 & 450 & 100.0 & 23.0 & 22.7 & 0.87 & 21.3 & 0.74 & 0.799 & 15.7 \\
$\mathbf{7}$ & 58 & 450 & 100.0 & 23.0 & 22.7 & 1.19 & 22.2 & 1.09 & 0.896 & 24.2 \\
$\mathbf{8}$ & 58 & 160 & 35.6 & 23.9 & 23.6 & 1.21 & 23.1 & 1.12 & 0.898 & 25.8 \\
$\mathbf{9}$ & 26 & 160 & 35.6 & 23.9 & 23.6 & 1.15 & 23.0 & 1.05 & 0.886 & 24.1 \\
$\mathbf{1 0}$ & 160 & 450 & 100.0 & 23.0 & 22.7 & 1.27 & 22.3 & 1.17 & 0.902 & 26.0 \\
\hline
\end{tabular}

(\#) Adopted short circuit current densities as obtained in (Cai et al. 2015). Observation: In the simplified BeerLambert model (Appendix B.1.1), the $J_{s c}$ is to some minute extent lower than $q G_{\max }$, as used in the setup of this model (see Matlab ${ }^{\mathrm{TM}}$ program in Supplementary Material), an effect which disappears for large values of $m>10$ in equation (8). However, as in the present modeling an $m=4.5$ is adopted, a feeble reduction of the $J_{s c}$, in comparison to $q G_{\max }$, is obtained. A meliorated model of the short circuit current density would, therefore, result in slightly higher maximal efficiencies as here presented, because of the higher resulting $J_{s c}$ values. 
Table 5

Relative increase or decrease of different performance parameters in the comparison of several suggested design proposals.

\begin{tabular}{|c|c|c|c|c|c|c|c|}
\hline Row & Design improvements & $\begin{array}{c}J_{S C} \\
{[\%]}\end{array}$ & $\begin{array}{c}V_{O C} \\
{[\%]}\end{array}$ & $\begin{array}{r}\boldsymbol{J}_{M P P} \\
{[\%]}\end{array}$ & $\begin{array}{r}V_{M P P} \\
{[\%]}\end{array}$ & $\begin{array}{c}\mathrm{FF} \\
{[\%]}\end{array}$ & $\begin{array}{r}\text { PCE } \\
{[\%]}\end{array}$ \\
\hline 1 & Sun - Design 9 & 4.0 & 32.8 & 8.3 & 41.2 & 10.8 & 53.0 \\
\hline 2 & Sun - Design 7 & 0.1 & 36.9 & 4.5 & 47.0 & 12.1 & 53.6 \\
\hline 3 & Sun - Design 10 & 0.1 & 46.0 & 4.7 & 57.5 & 12.8 & 64.8 \\
\hline 4 & Sun - Design 3 & 4.0 & 49.2 & 8.8 & 61.2 & 13.0 & 75.3 \\
\hline 5 & Sun - Design 8 & 4.0 & 40.1 & 8.6 & 50.7 & 12.3 & 63.7 \\
\hline 6 & Design 7 - Design 8 & 3.9 & 2.3 & 4.0 & 2.5 & 0.2 & 6.6 \\
\hline 7 & Design 9 - Design 8 & 0.0 & 5.5 & 0.3 & 6.7 & 1.4 & 7.0 \\
\hline 8 & Design 9 - Design 7 & -3.8 & 3.1 & -3.5 & 4.1 & 1.2 & 0.4 \\
\hline 9 & Design 7 - Design 10 & 0.0 & 6.6 & 0.2 & 7.1 & 0.6 & 7.3 \\
\hline 10 & Design 8 - Design 10 & -3.8 & 4.2 & -3.7 & 4.5 & 0.4 & 0.7 \\
\hline 11 & Design 10 - Design 3 & 3.9 & 2.2 & 4.0 & 2.3 & 0.2 & 6.4 \\
\hline 12 & Design 8 - Design 3 & 0.0 & 6.5 & 0.1 & 6.9 & 0.6 & 7.1 \\
\hline 13 & Design 9 - Design 3 & 0.0 & 12.4 & 0.4 & 14.1 & 2.0 & 14.6 \\
\hline 14 & Design 7 - Design 3 & 3.9 & 9.0 & 4.1 & 9.6 & 0.8 & 14.1 \\
\hline
\end{tabular}

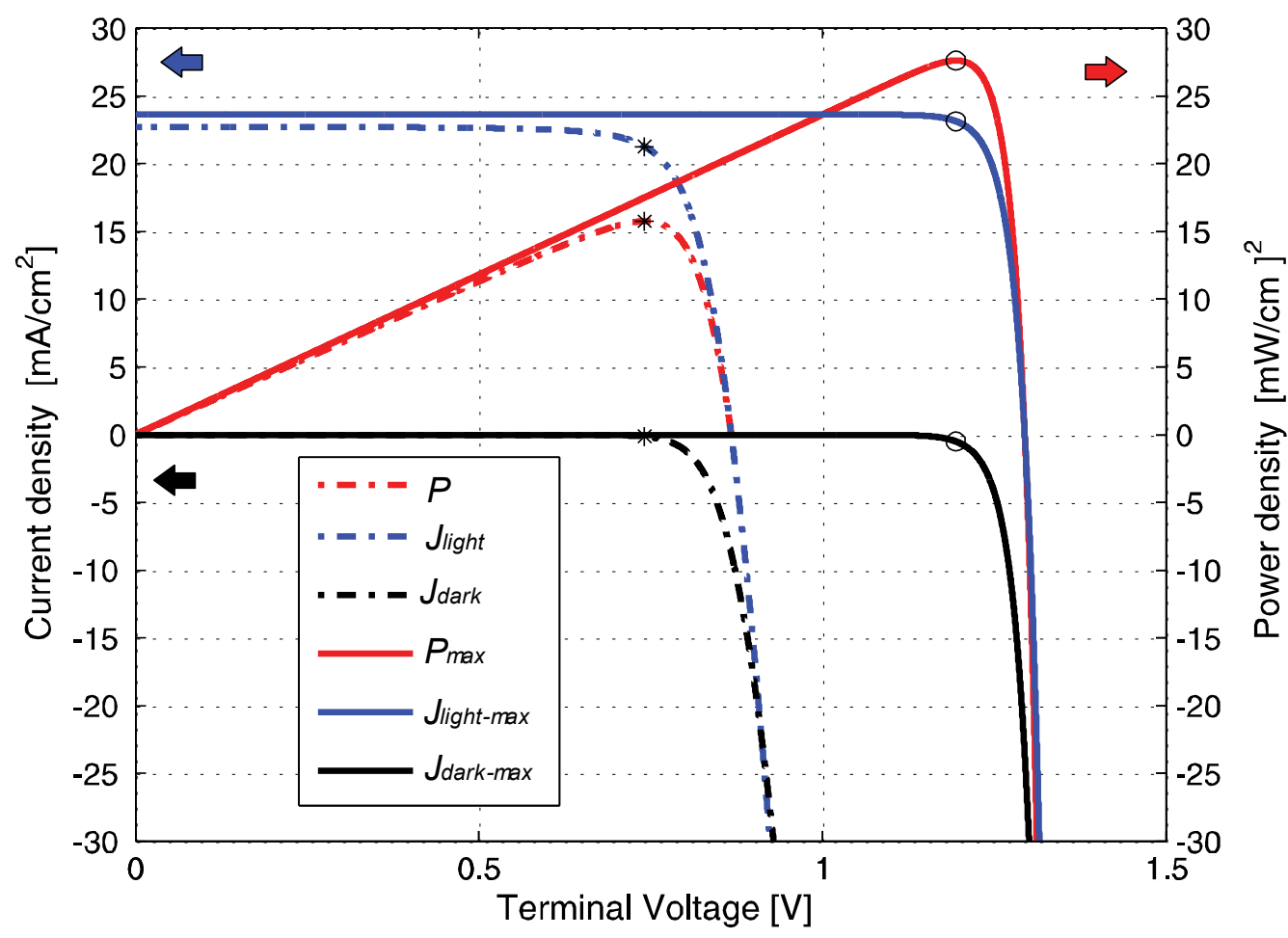

Fig. 5. J-V and power curves of the initial and optimized PSCs, with marked maximal power point (MPP) values: (i) curves for the solar cell configuration of the manufactured and modeled PSC in (Sun et al. 2015) ( $J_{\text {light }}, J_{\text {dark }}, P$ ) and (ii) curves for the simulated solar cell model, as show for a multidimensional optimization, with an maximal PCE of $27.6 \%$ for $f_{B}=160, t_{0}=160,\left(J_{\text {light-max }}, J_{\text {dark-max }}\right.$, $\left.P_{\max }\right)$ for AM 1.5 reference solar irradiance of $100 \mathrm{~mW} / \mathrm{cm}^{2}$. Stars and circles show the MPP operation points of these curves with MPP power densities of $15.7 \mathrm{~mW} / \mathrm{cm}^{2}$ and $27.6 \mathrm{~mW} / \mathrm{cm}^{2}$, corresponding in this normalized presentation to efficiencies of $\eta_{M P P}=$ $15.7 \%$ and $27.6 \%$ (Matlab $^{\mathrm{TM}}$ program for the configuration of this figure as a function of the cell's material properties in Supplementary Material). 
1 However, considering that the state-of-the-art cell is not based on light trapping (design 7); its PCE

2 can be increased by $6.6 \%$ using light trapping with spherical nanoparticles, as based on a $4 \%$ rise of

$3 J_{M P P}$ and a $2.5 \%$ rise of $V_{M P P}$, obtaining design 8 (Table 5 - row 6). A similar relative increase is

4 obtained for cells with improved $\left(f_{B} \geq 58\right.$, Fig. 3$)$ and perfect material properties $\left(f_{B}=160\right.$, Table 5 -

5 row 11). Further material property improvements in design 8 can raise the PCE by $7 \%$, as based on a

$6 \quad 6.9 \%$ rise of the $V_{M P P}$, obtaining cell design 3 (Table 5 - row 12). If no light trapping is considered at

7 all, the state-of-the-art efficiency (design 7) can be increased by $7.3 \%$, as almost only based on a

$8 \quad 7.1 \% V_{M P P}$ rise, obtaining cell design 10 (Table 5 - row 9), which presents the here considered most

9 ideal material properties with $f_{B}=160$. The efficiency of $26 \%$, as obtained by design $10\left(f_{B}=160\right)$,

10 can also be obtained with a much lower property improvement factor of $f_{B} \approx 62$, if light trapping is

11 used in a PSC with a $t_{0}=160 \mathrm{~nm}$ (horizontal dotted line in Fig. 3). Finally, the highest efficiency

12 increase (14.1\%) of design 7 is obtained, using light trapping and material property improvements

13 combined, resulting in design 3, as based on a $9.6 \%$ increase of the $V_{M P P}$ and a $4.1 \%$ increase of the

$14 J_{M P P}($ Table 5 - row 14).

15 Fig. 5 compares the two J-V curves, and its power curves, of (i) the cell as optimized in (Sun et al.,

16 2015) (dash-dotted curves), and (ii) the here considered, most ideal cell design. The latter was

17 obtained by a multidimensional optimization of a cell with spherical plasmonic nanoparticles in a

$18160 \mathrm{~nm}$ thick absorber layer (Table 3 - cell design 3). The PCE of this cell is only $0.2 \%$ lower in

19 comparison to the highest obtained efficiency in a cell with $t_{0}=400 \mathrm{~nm}$ (Fig. 3, Table 2). The

20 improvement of the cell's material properties and the light trapping with spherical nanoparticles

21 result in a significant PCE increase from $15.7 \%$ to $27.6 \%$.

\section{Discussions}

Summing up the nine one-dimensional efficiency increases, as presented in Table A.1, we register a total contribution of $4.5 \%$, for a boundary amplification factor of $f_{B}=160$. However, the combined contribution of these variables, in a multidimensional optimization, results with the maximum value 
1 of $27.6 \%$ (Table 3 ) in a much higher efficiency rise of $27.6 \%-15.7 \%=13.4 \%$, considering the same

$2 f_{B}$ factor. Consequently, because of the summed single contributions of the one-dimensional PCE

3 increases, the efficiency raises by $4.5 \%$, and because of further effects, a much higher growth, with

4 the value of $13.4 \%-4.5 \%=8.9 \%$ can be obtained. We registered a similar behavior also in the

5 comparison of one- and two-dimensional optimizations (Appendix, Section A.1) and assign this large

6 additional PCE increase to the nonlinearities, as inherent to the analyzed PSC and its drift-diffusion

7 model. As a result, higher-dimensional optimizations are much more effective, in comparison to the

8 state-of-the-art one- and two-dimensional optimizations.

9 The proper efficiency growth in Fig. 3 presents also a nonlinear behavior, as the highest PCE

10 gradients are obtained for low $f_{B}$ values, which correspond to small material property improvements

11 in the hypercube space. Hence, the multidimensional efficiency optimization is most effective for

12 cells, which efficiency is close, or lower than the state-of-the-art PCE with $f_{B} \approx 58$, as minor

13 modifications in the material properties, lead to the highest efficiency gradients. Light trapping

14 enhances this effectiveness in the multidimensional optimizations, because of the higher gradients of

15 the related curves in Fig. 3.

16 The highest and second-highest values of $J_{s c}$ and $J_{M P P}$ are obtained by the light trapping cell designs

175 and 4 , presenting the absorber layer thicknesses of $t_{0}=400$ and $320 \mathrm{~nm}$ (Table 4). Meanwhile, the

18 largest and second-largest values of the efficiencies are obtained by cell designs 5 and 3, presenting a

$19 t_{0}$ of 400 and $160 \mathrm{~nm}$. In the case of $V_{O C}$ and $V_{M P P}$, the highest values are surprisingly obtained for the

20 cell with the thinnest absorber layer (design 1), resulting inclusively to the largest FF, which means

21 that $V_{O C}$ and $V_{M P P}$ decrease proportionally with the increase of $t_{0}$ (Table 3). As a result of these

22 effects, thin absorber layers result in reasonable high efficiencies, obtaining thus the second-highest

23 PCE with the selected favorite cell, which presents an absorber layer of $t_{0}=160 \mathrm{~nm}($ design 3$)$.

24 We account the increases in $V_{0 C}$ and FF, on the concomitant (i) reduction of $t_{0}$, and (ii) the light

25 trapping effect, which result in its combination to high PCEs. The high $V_{O C}$ is a function of the

26 photon recycling effect, as inherent to PSCs (Pazos-Outón et al., 2016), which increases by light 
1 trapping (Sha et al., 2015 and Kirchartz et al., 2016), and results furthermore in a larger fill factor

2 (FF) (Sha et al., 2015). Thin absorber layers present lower recombination of charge carriers, as

3 discussed in (Domanski et al., 2016) and Appendix A.5. As a result, thin absorber layers increase the

$4 \quad$ FF and the $V_{o c}$, even without light trapping, as shown by numerical simulations in (Devi et al., 2018).

5 The lower absorber layer thicknesses can be manufactured with ultra-thin coating techniques (Liu, 6 2017), and the solvent-solvent extraction methods (Zhou et al., 2015), where the latter results in

7 smooth, and thickness adjustable absorber layers, in a large range of $t_{0}=20 \ldots 410 \mathrm{~nm}$.

8 PSCs with $t_{0} \approx 450 \mathrm{~nm}$ do already present a low $\mathrm{Pb}$ content, which is similar, to the heavy-metal

9 content of state-of-the-art silicon photovoltaic modules (Green et al., 2014; Stasiulionis, 2015).

10 Fortunately, the here presented thickness reduction of the absorber layer to $t_{0}=160 \mathrm{~nm}$ leads to an

11 additional reduction of the $\mathrm{Pb}$ residual.

12 The here used modeling of the short circuit current density presents high accuracies for the cases: (i)

13 without light trapping, and (ii) light trapping with spherical nanoparticles; because the setup of the

14 short circuit current density is based on high-resolution simulations, such as (i) the OTM modeling

15 (Sun et al., 2015), and (ii) the FDTD modeling (Cai et al., 2015) (Appendix section B.1.4).

16 Furthermore, we consider reasonable low uncertainties in the Beer-Lambert modeling (Appendix

17 B.1.1 to B.1.3). Additionally, we remark that large deviations in $\lambda_{\text {ave }}$ value result in very small

18 efficiency deviations for the same $f_{B}$ (Tables 1 to 3 ). Therefore, even under a hypothetical

19 consideration of large uncertainties in the Beer-Lambert law, and its related $\lambda_{\text {ave, }}$ only small

20 uncertainties of the optimized efficiency values would be obtained.

21 The highest here achieved efficiency for single-junction PSCs of 27.76\% (Table 2), is higher than (i)

22 the state-of-the-art efficiency of $24.2 \%$ and (ii) the highest simulated efficiency of 25\% (Agarwal

23 and Nair, 2014, 2015). In fact, a large set of our optimized design proposals, as presented in Fig. 3,

24 increase the state-of-the-art PCEs of manufactured and simulated solar cells. As expected, all

25 proposals present a lower efficiency than (i) the lowest value of the theoretical upper limit with PCE 
$1=29.9 \%$, as considered for zero surface recombination velocities and ideal light trapping (Ren et al.,

2 2017).

3 The material property improvements as selected by the multidimensional optimization algorithm are

4 general and must not coincide with property values of manufactured PSCs, as improvements of the

5 different properties can appear at individual scales in those cells. We used the general property

6 improvement, as expressed by $f_{B}$ in order to: (i) illustrate the large efficiency advantage of

7 multidimensional property improvements; (ii) present high- and highest-performance cell designs with low $\mathrm{Pb}$ content, and discuss by which techniques these cells can be manufactured; (iii) estimate and discuss the percentual improvements of several performance parameters, comparing cells in different development states, or cell designs; (iv) identify dependencies in-between model variables,

11 as appeared between $t_{0}$ and $\lambda_{\text {ave }}$ (Table A.2 and Figs. 2a and 2b); (v) present a complete set of 12 possible two-dimensional sensitivity analyses (Appendix A.1); and (vi) identified from the latter 13 analyses that $s_{f}, s_{b}, t_{0}, \lambda_{\text {ave }}$ and $D_{p}$ are the most important optimization variables, in this order. As a 14 result, we get a better understanding and provide useful knowledge for the efficiency optimization of 15 manufactured PSCs. The presented results can be used as a roadmap, showing to which extend a PSC's performance parameters and its efficiency can be increased, by different measures and in several development states, while its $\mathrm{Pb}$ content is reduced. In our design proposals, we consider one, two (Table A.1), and multidimensional improvements of the cell's material properties (Fig. 3); which

19 are combined with light trapping, and thin-film coating techniques.

20 Whereas material property improvements lead to the highest relative efficiency increase in cells, 21 which PCE is less or equal to the state-of-the-art efficiency (Fig. 3, Table 5 - row 2), light trapping techniques are most efficient to improve the efficiency in cells, which PCE is higher or equal than 23 the state-of-the-art PCE (Fig. 3, Table 5 - rows 6 and 11). While the former is almost solely based on $24 V_{M P P}$ increase, the latter increase both, the $J_{M P P}$ and the $V_{M P P}$. Being the state-of-the-art efficiency 25 based on light trapping (cell design 9) or not (cell design 7), the cell's efficiency can be increased by: 
1 (i) further material property improvements (Table 5 - row 13), and (ii) its combination with light

2 trapping (Table 5 - row 14), resulting in both cases to a PCE increase of approximately 14\%.

3 The $\lambda_{\text {ave }}$ should be always determined as a function of $t_{0}$, being this in an $m$-constraint or a $f_{B^{-}}$

4 constrained relationship, where the former is simply to calculate, and the results in slightly higher

5 efficiency values (Table 1 to 3 - last two columns), if very low reflection losses are configurable. As

6 the remaining properties stand not in a relationship, the here presented multidimensional

7 optimizations can be substituted by simple model simulations, in cell manufacturing and research,

8 adopting the two-dimensional sensitivity analysis, as presented in Fig. 2f; and alternatively, using the

9 constraint condition in equation (9). We suggest that PSC manufacturing should be based on

10 thickness adjustable thin-film coating techniques (Zhou et al., 2015, Liu, 2017), where an ideal to can

11 be adjusted as a function of a configured $\lambda_{\text {ave. }}$ Otherwise, adjustable light trapping techniques (Cai et

12 al., 2015) may be used to configure an adjustable ideal $\lambda_{\text {ave, }}$ for a fixed absorber layer thickness. We

13 also like to advise that some solar cell types have an ideal built-in field (Green, 2009), as related to

14 an ideal built-in voltage, an effect that was not observed with the present model in the constraint of

$15 \quad V_{b i} \leq 1.4 \mathrm{~V}$.

16

\section{Conclusions}

18 Our analyses lead to a better understanding of the cell's optimization process, and we propose high-

19 efficiency PSC designs with ultra-thin absorber layers with a significant lower Pb content. The high

20 PCE values for the absorber layer thickness of $160 \mathrm{~nm}$, are obtained as light trapping shows similar

21 current densities for a wide range of absorber layer thicknesses, and as $V_{O C}$ and $V_{M P P}$ increase as a

22 function of the thickness reduction of the absorber layer. We demonstrate and discuss a large

23 efficiency advantage, in multidimensional optimizations, and assign this effect to the nonlinear

24 behavior of the drift-diffusion model of electrons and holes. As discussed, our optimizations result in

25 PCE values, which appear in expected, and reasonable, ranges of the efficiency, and we consider low

26 uncertainties for simulated design proposals. We obtained high sensitivities for low $f_{B}$ values, and 
1 these sensitivities improve with light trapping. Furthermore, light trapping is most efficient for high

$2 f_{B}$ values, which result in the highest PCE increases. Therefore, light trapping is always

3 advantageous. For high $f_{B}$ factors, as it leads to the highest PCE rises, and for low $f_{B}$ factors, as it not

4 only increases the efficiency but also helps to improve the sensitivity of the further model variables.

5 However, researchers which like to optimize PSCs with efficiencies much lower than the state-of-

6 the-art PCE should focus principally on the improvement of the material properties, by reason of the

7 resulting high PCE gradients in this range.

8

\section{$9 \quad$ Future works}

10 Further FDTD simulations for cells with shape optimized nanoparticles (Appendix sections A.2 and

11 B.1.5) and special cell designs with high $J_{s c}$ values (Appendix section A.6 and Table A.4) should be

12 accomplished. A protocol for the multidimensional material property measurement and control

13 should be developed in the research with the manufactured prototypes. 


\begin{tabular}{|c|c|}
\hline$D_{n}$ & Diffusion coefficient of electrons $\left[\mathrm{cm}^{2} / \mathrm{s}\right]$, \\
\hline$D_{p}$ & Diffusion coefficient of holes $\left[\mathrm{cm}^{2} / \mathrm{s}\right]$, \\
\hline$f_{B}$ & Boundary expansion factor $[-]$, \\
\hline$G_{A M 1.5}$ & Solar irradiance with air mass $1.5\left[\mathrm{~mW} / \mathrm{cm}^{2}\right]$ \\
\hline$G(x)$ & Generation rate of charges as a function of $x\left[\mathrm{~s}^{-1} \mathrm{~cm}^{-3}\right]$, \\
\hline$G_{e f f}$ & Effective charge carrier generation $\left[\mathrm{s}^{-1} \mathrm{~cm}^{-3}\right]$, \\
\hline$G_{\max }$ & Maximal or total charge carrier generation $\left[\mathrm{s}^{-1} \mathrm{~m}^{-2}\right]$ \\
\hline$J_{b}$ & $\begin{array}{l}\text { Electron recombination current density of the back charge } \\
\text { conduction layer at } x=t_{0}\left[\mathrm{~mA} / \mathrm{cm}^{2}\right]\end{array}$ \\
\hline$J_{\text {dark }}$ & Measurable current density in the dark $\left[\mathrm{mA} / \mathrm{cm}^{2}\right]$, \\
\hline$J_{f}$ & $\begin{array}{l}\text { Electron recombination current density of the front charge } \\
\text { conduction layer at } x=O\left[\mathrm{~mA} / \mathrm{cm}^{2}\right]\end{array}$ \\
\hline$J_{\text {light }}$ & $\begin{array}{l}\text { The measurable current density under light exposure } \\
{\left[\mathrm{mA} / \mathrm{cm}^{2}\right]}\end{array}$ \\
\hline$J_{M P P, i}$ & Maximal power point current density $\left[\mathrm{mA} / \mathrm{cm}^{2}\right]$, \\
\hline$J_{n}$ & $\begin{array}{l}\text { Electron current density at the back charge conduction layer } \\
{\left[\mathrm{mA} / \mathrm{cm}^{2}\right],}\end{array}$ \\
\hline$J_{p}$ & $\begin{array}{l}\text { Hole current density at the front charge conduction layer } \\
{\left[\mathrm{mA} / \mathrm{cm}^{2}\right]}\end{array}$ \\
\hline$J_{s c}$ & Short circuit current density $\left[\mathrm{mA} / \mathrm{cm}^{2}\right]$, \\
\hline$m$ & Factor which relates $t_{0}$ and $\lambda_{\text {ave }}$ in the Beer-Lambert law [-], \\
\hline$P_{M P P}$ & Maximal power point power density $\left[\mathrm{mW} / \mathrm{cm}^{2}\right]$, \\
\hline$k_{B}$ & Boltzmann constant $1.38064852 \times 10^{-23}[\mathrm{~J} / \mathrm{K}]$ \\
\hline$q$ & Electric charge of an electron or hole $[\mathrm{mAs}]$ \\
\hline$s f$ & Surface recombination velocity of electrons $\left(s_{n}\right)[\mathrm{cm} / \mathrm{s}]$, \\
\hline$s b$ & Surface recombination velocity of holes $\left(s_{p}\right)[\mathrm{cm} / \mathrm{s}]$ \\
\hline$T$ & Cell temperature $[\mathrm{K}]$, \\
\hline$t_{0}$ & Absorber layer thickness [nm], \\
\hline$t_{0-\min }$ & Minimal absorber layer thickness [nm], \\
\hline to-min & Minimal necessary absorber layer thickness [nm], \\
\hline$V$ & Terminal voltage of the solar cell [V], \\
\hline$V_{b i}$ & Built-in voltage [V], \\
\hline$V_{M P P}$ & Maximal power point voltage $[\mathrm{V}]$, \\
\hline
\end{tabular}




$\begin{array}{ll}V_{o c} & \text { Open-circuit voltage }[\mathrm{V}], \\ x=0 \ldots t_{0} & \text { Solar irradiance penetration depth }[\mathrm{m}], \\ X_{j}=X_{1} \ldots X_{9} & X_{j} \text { is one of the nine model variables of the PSC model, } \\ X_{j, m e} & \text { A model variable extracted from the measured J-V curve, } X_{j}, \\ & \text { is one of the nine model variables of the PSC model, } \\ X_{j-\min } \ldots X_{j, \max } & \text { Variable expansion range for the variable j, }\end{array}$

\section{Greek symbols}

$\Delta n \quad$ Excess minority carrier concentration of electrons $\left[\mathrm{cm}^{-3}\right]$,

$\Delta p \quad$ Excess minority carrier concentration of holes $\left[\mathrm{cm}^{-3}\right]$,

$\eta_{i} \quad$ Optimized PCE for the i-th optimization iteration [\%],

$\eta_{\max } \quad$ Optimized efficiency value [\%],

$\lambda \quad$ Wavelength of the solar irradiance $[\mathrm{nm}]$,

$\lambda_{\text {ave }} \quad$ Average optical decay length [nm].

$\mu_{n} \quad$ Drift coefficient or mobility of electrons $\left[\mathrm{cm}^{2} \mathrm{~V}^{-1} \mathrm{~s}^{-1}\right]$

$\mu_{p} \quad$ Drift coefficient or mobility of holes $\left[\mathrm{cm}^{2} \mathrm{~V}^{-1} \mathrm{~s}^{-1}\right]$

\section{Indices}

$i=1 \ldots N \quad$ Iterations in the optimization of the efficiency,

$k=1 \ldots M \quad$ Iterations in the optimization of the power curve,

$j=1 \ldots 9 \quad$ Index, which counts the nine variables,

\section{Declaration of Competing Interests}

This manuscript does not have any conflict of interest.

\section{Acknowledgments}

The authors thank (i) Comissão de Aperfeiçoamento de Pessoal do Nível Superior (CAPES), (ii) Fotovoltaica UFSC - Grupo de Pesquisa Estratégica em Energia Solar at Universidade Federal de Santa Catarina and (iii) Agência Nacional de Energia Elétrica (ANNEL) for their financial support.

\section{Appendix}

The appendix and supporting material associated with this article can be found, on the online version: 
Adinolfi, V., Peng, W., Walters, G., Bakr, O.M., Sargent, E.H., 2017. The electrical and optical properties of organometal halide perovskites relevant to optoelectronic performance. Advanced Materials, 1-13.

Agarwal, S., Nair, P.R., 2014. Performance optimization for Perovskite based solar cells, Proceedings of the 40th IEEE Photovoltaic Specialist Conference (PVSC). Colorado, pp. 15151518.

Agarwal, S., Nair, P.R., 2015. Device engineering of perovskite solar cells to achieve near ideal efficiency. Applied Physics Letters 107(12), 1-5.

Algora, C., Díaz, V., 2000. Influence of series resistance on guidelines for manufacture of concentrator p-on-n GaAs solar cells. Progress in Photovoltaics: Research and Applications $8(2), 211-225$.

Amu, T.L., 2014. Performance optimization of tin halide perovskite solar cells via numerical simulation, (Master thesis). African University of Science and Technology, Abuja, Nigeria.

Atwater, H.A., Polman, A., 2010. Plasmonics for improved photovoltaic devices. Nature materials 9(3), 205-213.

Azri, F., Meftah, A., Sengouga, N., Meftah, A., 2019. Electron and hole transport layers optimization by numerical simulation of a perovskite solar cell. Solar Energy 181, 372-378.

Ball, J.M., Stranks, S.D., Hörantner, M.T., Hüttner, S., Zhang, W., Crossland, E.J., Ramirez, I., Riede, M., Johnston, M.B., Friend, R.H., 2015. Optical properties and limiting photocurrent of thin-film perovskite solar cells. Energy \& Environmental Science 8(2), 602-609.

Bazzo, T.d.P.M., Kölzer, J.F., Carlson, R., Wurtz, F., Gerbaud, L., 2017. Multiphysics design optimization of a permanent magnet synchronous generator. IEEE Transactions on Industrial Electronics 64(12), 9815-9823.

Brittman, S., Adhyaksa, G.W.P., Garnett, E.C., 2015. The expanding world of hybrid perovskites: Materials properties and emerging applications. MRS Communications 5(01), 7-26.

Burschka, J., Pellet, N., Moon, S.-J., Humphry-Baker, R., Gao, P., Nazeeruddin, M.K., Grätzel, M., 2013. Sequential deposition as a route to high-performance perovskite-sensitized solar cells. Nature 499(7458), 316-319.

Byrd, R.H., Hribar, M.E., Nocedal, J., 1999. An interior point algorithm for large-scale nonlinear programming. SIAM Journal on Optimization 9(4), 877-900.

Cai, B., Peng, Y., Cheng, Y.-B., Gu, M., 2015. 4-fold photocurrent enhancement in ultrathin nanoplasmonic perovskite solar cells. Optics Express 23(24), A1700-A1706.

Chowdhury, M., Alam, M., 2014. A physics-based analytical model for bulk heterojunction organic solar cells incorporating monomolecular recombination mechanism. Current Applied Physics 14(3), 340-344.

Crow, M.L., 2009. Computational methods for electric power systems. Crc Press.

Devi, C., Mehra, R., 2019. Device simulation of lead-free MASnI 3 solar cell with $\mathrm{CuSbS}_{2}$ (copper antimony sulfide). Journal of Materials Science 54(7), 5615-5624.

Devi, N., Parrey, K.A., Aziz, A., Datta, S., 2018. Numerical simulations of perovskite thin-film solar cells using a CdS hole blocking layer. Journal of Vacuum Science \& Technology B, Nanotechnology and Microelectronics: Materials, Processing, Measurement, and Phenomena 36(4), 04G105.

Dixit, H., Punetha, D., Pandey, S.K., 2019. Improvement in performance of lead free inverted perovskite solar cell by optimization of solar parameters. Optik 179, 969-976.

Domanski, K., Correa-Baena, J.-P., Mine, N., Nazeeruddin, M.K., Abate, A., Saliba, M., Tress, W., Hagfeldt, A., Grätzel, M., 2016. Not all that glitters is gold: Metal-migration-induced degradation in perovskite solar cells. ACS Nano 10(6), 6306-6314.

Foster, J.M., Snaith, H.J., Leijtens, T., Richardson, G., 2014. A model for the operation of perovskite based hybrid solar cells: Formulation, analysis, and comparison to experiment. SIAM Journal on Applied Mathematics 74(6), 1935-1966. 
Green, M., A., 2009. Do built-in fields improve solar cell performance? Progress in Photovoltaics: Research and Applications 17(1), 57-66.

Green, M.A., Ho-Baillie, A., Snaith, H.J., 2014. The emergence of perovskite solar cells. Nature Photonics 8(7), 506-514.

Huang, H., Huang, J., 2014. Organic and hybrid solar cells, first ed. Springer, New York.

Iftiquar, S.M., Yi, J., 2016. Numerical simulation and light trapping in perovskite solar cell. Journal of Photonics for Energy 6(2), 1-10.

Jiang, Y., Green, M.A., Sheng, R., Ho-Baillie, A., 2015. Room temperature optical properties of organic-inorganic lead halide perovskites. Solar Energy Materials and Solar Cells 137, 253257.

Jung, E.H., Jeon, N.J., Park, E.Y., Moon, C.S., Shin, T.J., Yang, T.-Y., Noh, J.H., Seo, J., 2019. Efficient, stable and scalable perovskite solar cells using poly (3-hexylthiophene). Nature 567(7749), 511.

Kakavelakis, G., Petridis, K., Kymakis, E., 2017. Recent advances in plasmonic metal and rare-earthelement upconversion nanoparticle doped perovskite solar cells. Journal of Materials Chemistry A 5(41), 21604-21624.

Kanoun, A.-A., Kanoun, M.B., Merad, A.E., Goumri-Said, S., 2019. Toward development of highperformance perovskite solar cells based on $\mathrm{CH} 3 \mathrm{NH} 3 \mathrm{GeI} 3$ using computational approach. Solar Energy 182, 237-244.

Kim, H.D., Ohkita, H., 2017. Potential Improvement in Fill Factor of Lead-Halide Perovskite Solar Cells. Solar RRL 1(6), 1-6.

Kirchartz, T., Staub, F., Rau, U., 2016. Impact of photon recycling on the open-circuit voltage of metal halide perovskite solar cells. ACS Energy Letters 1(4), 731-739.

Kojima, A., Teshima, K., Shirai, Y., Miyasaka, T., 2009. Organometal halide perovskites as visiblelight sensitizers for photovoltaic cells. Journal of the American Chemical Society 131(17), 6050-6051.

Kratzenberg, M., Rambo, C., Rüther, R., Beyer, H., 2015. Sensitivity analyses of a p-i-n perovskite solar cell with a fixed band gap, in: Society, I.S.E. (Ed.) Solar World Congress. Daegu, Korea, pp. 1-5.

Liu, C., Li, W., Fan, J., Mai, Y., 2018. A brief review on the lead element substitution in perovskite solar cells. Journal of Energy Chemistry 27(4), 1054-1066.

Liu, F., Zhu, J., Wei, J., Li, Y., Lv, M., Yang, S., Zhang, B., Yao, J., Dai, S., 2014. Numerical simulation: toward the design of high-efficiency planar perovskite solar cells. Applied Physics Letters 104(25), 1-4.

Liu, Y., 2017. Plasmonic metal nanoparticle films for solar cells with ultra-thin absorber layers: Low temperature synthesis and application. (Doctoral thesis), Freie Universität, Berlin, Germany.

Martynov, Y.B., Nazmitdinov, R.G., Moià-Pol, A., Gladyshev, P.P., Tameev, A.R., Vannikov, A.V., Pudlak, M., 2017. On the efficiency limit of $\mathrm{ZnO} / \mathrm{CH}_{3} \mathrm{NH}_{3} \mathrm{PbI}_{3} / \mathrm{CuI}$ perovskite solar cells. Physical Chemistry Chemical Physics 19(30), 19916-19921.

NREL, 2019. Best Research-Cell Efficiencies, National Renewable Energy Laboratory Photovoltaic Research Hompage.

O’Regan, B., Grätzel, M., 1991. A low-cost, high-efficiency solar cell based on dye-sensitized colloidal $\mathrm{TiO}_{2}$ films. Nature 353(6346), 737-740.

Park, J.-K., Kang, J.-C., Kim, S.Y., Son, B.H., Park, J.-Y., Lee, S., Ahn, Y.H., 2012. Diffusion length in nanoporous photoelectrodes of dye-sensitized solar cells under operating conditions measured by photocurrent microscopy. The Journal of Physical Chemistry Letters 3(23), 36323638.

Pazos-Outón, L.M., Szumilo, M., Lamboll, R., Richter, J.M., Crespo-Quesada, M., Abdi-Jalebi, M., Beeson, H.J., Vrućinić, M., Alsari, M., Snaith, H.J., 2016. Photon recycling in lead iodide perovskite solar cells. Science 351(6280), 1430-1433. 
Qiu, W., Merckx, T., Jaysankar, M., de la Huerta, C.M., Rakocevic, L., Zhang, W., Paetzold, U., Gehlhaar, R., Froyen, L., Poortmans, J., 2016. Pinhole-free perovskite films for efficient solar modules. Energy \& Environmental Science 9(2), 484-489.

Ren, X., Wang, Z., Sha, W.E., Choy, W.C., 2017. Exploring the way to approach the efficiency limit of perovskite solar cells by drift-diffusion model. Acs Photonics 4(4), 934-942.

Rong, Y., Hou, X., Hu, Y., Mei, A., Liu, L., Wang, P., Han, H., 2017. Synergy of ammonium chloride and moisture on perovskite crystallization for efficient printable mesoscopic solar cells. Nature Communications 8, 1-8.

Rühle, S., 2016. Tabulated values of the Shockley-Queisser limit for single junction solar cells. Solar Energy 130, 139-147.

Rüther, R., Kleiss, G., Reiche, K., 2002. Spectral effects on amorphous silicon solar module fill factors. Solar Energy Materials and Solar Cells 71(3), 375-385.

Sha, W.E., Ren, X., Chen, L., Choy, W.C., 2015. The efficiency limit of $\mathrm{CH}_{3} \mathrm{NH}_{3} \mathrm{PbI}_{3}$ perovskite solar cells. Applied Physics Letters 106(22), 1-5.

Shockley, W., Queisser, H.J., 1961. Detailed balance limit of efficiency of p-n junction solar cells. Journal of Applied Physics 32(3), 510-519.

Stasiulionis, J., 2015. Life cycle assessment of perovskite solar cells and comparison to silicon solar cells, (Master thesis). Chalmers University of Technology, Sweden, p. 50.

Sun, X., Asadpour, R., Nie, W., Mohite, A.D., Alam, M.A., 2015. A physics-based analytical model for perovskite solar cells. IEEE Journal of Photovoltaics 5(5), 1389-1394.

Taretto, K., Soldera, M., Koffman-Frischknecht, A., 2017. Material Parameters and Perspectives for Efficiency Improvements in Perovskite Solar Cells Obtained by Analytical Modeling. IEEE Journal of Photovoltaics 7(1), 206-213.

Wong, W.-Y., Wang, X.-Z., He, Z., Djurišić, A.B., Yip, C.-T., Cheung, K.-Y., Wang, H., Mak, C.S.K., Chan, W.-K., 2007. Metallated conjugated polymers as a new avenue towards highefficiency polymer solar cells. Nature Materials 6(7), 521-527.

Yin, W.-J., Shi, T., Yan, Y., 2014. Unique properties of halide perovskites as possible origins of the superior solar cell performance. Advanced Materials 26(27), 4653-4658.

Zandi, S., Razaghi, M., 2019. Finite element simulation of perovskite solar cell: A study on efficiency improvement based on structural and material modification. Solar Energy 179, 298306.

Zhao, P., Liu, Z., Lin, Z., Chen, D., Su, J., Zhang, C., Zhang, J., Chang, J., Hao, Y., 2018. Device simulation of inverted $\mathrm{CH} 3 \mathrm{NH} 3 \mathrm{PbI} 3-\mathrm{x} \mathrm{Cl}$ x perovskite solar cells based on PCBM electron transport layer and NiO hole transport layer. Solar Energy 169, 11-18.

Zhou, Y., Yang, M., Wu, W., Vasiliev, A.L., Zhu, K., Padture, N.P., 2015. Room-temperature crystallization of hybrid-perovskite thin films via solvent-solvent extraction for highperformance solar cells. Journal of Materials Chemistry A 3(15), 8178-8184. 